\title{
BOUNDARY STABILIZATION OF QUASILINEAR HYPERBOLIC SYSTEMS OF BALANCE LAWS: EXPONENTIAL DECAY FOR SMALL SOURCE TERMS
}

\author{
MARTIN GUGAT, VINCENT PERROLLAZ, AND LIONEL ROSIER
}

\begin{abstract}
We investigate the long-time behavior of solutions of quasilinear hyperbolic systems with transparent boundary conditions when small source terms are incorporated in the system. Even if the finite-time stability of the system is not preserved, it is shown here that an exponential convergence towards the steady state still holds with a decay rate which is proportional to the logarithm of the amplitude of the source term. The result is stated for a system with dynamical boundary conditions in order to deal with initial data that are free of any compatibility condition.
\end{abstract}

\section{INTRODUCTION}

Solutions of certain hyperbolic systems can reach the equilibrium state in finite time. Such a property, called finite-time stability in [1, 17, 18] or super-stability in [19], was first noticed in $[12,15]$ for the (linear) wave equation. The extension of such a property to the wave equation on networks was addressed in [1, 19].

Fortunately, the finite-time stability still occurs for systems of $2 \times 2$ quasilinear hyperbolic equations of diagonal form without source terms, as it was noticed in [13] with initial data satisfying some compatibility conditions to prevent the emergence of shockwaves, and next in $[17,18]$ for arbitrary initial data by replacing homogeneous boundary conditions by some dynamical boundary conditions.

The finite-time stabilization of a quasilinear hyperbolic system with source terms seems to be very challenging. In [4], the authors proved that a $2 \times 2$ linear hyperbolic system with source terms can be stabilized to the origin in finite-time by using some boundary feedback laws designed with the backstepping approach.

On the other hand, the finite-time stability of a system may be lost when a small, bounded perturbation is added to the system. A famous example is provided by the telegraph equation

$$
\begin{aligned}
\partial_{t}^{2} y-\partial_{x}^{2} y+\epsilon \partial_{t} y & =0, \quad(t, x) \in(0,+\infty) \times(0, L), \\
y(t, 0)=0, \quad y_{x}(t, L) & =-y_{t}(t, L) .
\end{aligned}
$$

However, as it was noticed in [8] for (1.1)-(1.2) or more generally for a nonlinear perturbation of the wave equation, the exponential stability of the system is preserved, with a decay rate proportional to $\ln \left(\epsilon^{-1}\right)$. See also [11] for the exponential stabilization of the isothermal Euler

2000 Mathematics Subject Classification. 35L50,35L60,76B75,93D15.

Key words and phrases. System of balance laws, shallow water equations, telegraph equation, finite-time stability, dynamical boundary conditions, exponential stability, decay rate. 
equations and [5] for the loss of the stability when incorporating an arbitrarily small delay in a transparent boundary condition for the wave equation.

The aim of this paper is to show that the robustness property noticed in [8] is shared by most of the finite-time stable systems. The first result in this paper shows that a linear finite-time stable system with a (small) disturbance is exponentially stable with a decay rate proportional to the logarithm of the amplitude of the perturbation. We refer the reader to [21, Theorem 4.2] for a sufficient condition involving the resolvent for the finite-time stability of a linear system.

Theorem 1. Let $A$ be an operator generating a strongly continuous semigroup $\left(e^{t A}\right)_{t \geq 0}$ in an Hilbert space $H$, and let $B \in \mathcal{L}(H)$ be a bounded operator. Assume that $e^{T A}=0$ for some $T>0$. Then there exist some positive numbers $\epsilon_{0}, M, C$ such that for any $\epsilon \in\left(0, \epsilon_{0}\right)$, it holds

$$
\left\|e^{t(A+\epsilon B)}\right\|_{\mathcal{L}(H)} \leq M \inf \left(1, \frac{e^{-\left(C \ln \epsilon^{-1}\right) t}}{\epsilon}\right) \quad \forall t \geq 0 .
$$

The (simple) proof of Theorem 1 is given in Appendix. It rests on the observation that the solution of a Cauchy problem can be obtained as a fixed-point of a map, derived from Duhamel formula, in a weighted space. The weight is related to the decay rate. It is unclear whether such an approach could be extended to quasilinear systems.

It should be noticed that the estimate in (1.3) is essentially sharp. Indeed, for the system

$$
\begin{cases}\partial_{t} u+c \partial_{x} u=\epsilon v, & x \in(0, L), t>0, \\ \partial_{t} v-c \partial_{x} v=\epsilon u, & x \in(0, L), t>0, \\ u(t, 0)=v(t, L)=0, & t>0, \\ u(0, x)=u_{0}(x), \quad v(0, x)=v_{0}(x), & x \in(0, L),\end{cases}
$$

we shall prove that the decay rate is roughly speaking bounded from below by $(c / L) \ln \epsilon^{-1}$.

Theorem 2. Let $c>0$, let $A(u, v):=\left(-c \partial_{x} u, c \partial_{x} v\right)$ be the operator with domain

$$
D(A):=\left\{(u, v) \in\left[H^{1}(0, L)\right]^{2} ; u(0)=v(L)=0\right\} \subset H:=\left[L^{2}(0, L)\right]^{2},
$$

and let $B(u, v):=(v, u)$. Then for any $\kappa>c / L$, there exist some numbers $K, \epsilon_{0}>0$ such that for any $\epsilon \in\left(0, \epsilon_{0}\right)$, it holds

$$
\left\|e^{t(A+\epsilon B)}\right\|_{\mathcal{L}(H)} \geq K e^{-\left(\kappa \ln \epsilon^{-1}\right) t} \quad \forall t \geq 0 .
$$

The main aim of the paper is to investigate the application of transparent boundary conditions, or more generally of dynamical boundary conditions as in [17, 18], to $2 \times 2$ quasilinear hyperbolic systems in diagonal form with small source terms

$$
\begin{aligned}
\partial_{t} u+\lambda(u, v) \partial_{x} u & =\epsilon f(u, v), \\
\partial_{t} v-\mu(u, v) \partial_{x} v & =\epsilon g(u, v)
\end{aligned}
$$

where $\lambda(u, v)>c, \mu(u, v)>c$ for some constant $c>0$, and $0<\epsilon \ll 1$. Our results are stated when $f$ and $g$ do not depend on $\epsilon$, but there are still valid when $f$ and $g$ depend on $\epsilon$ but are bounded in $W^{2, \infty}(0, L)$ for $0<\epsilon \ll 1$.

We shall prove that for $\epsilon$ small enough and for initial data sufficiently close to a steady state of (1.6)-(1.7), the solution of (1.6)-(1.7) with dynamical boundary conditions converge exponentially to the steady state with a decay rate proportional to $\ln \left(\epsilon^{-1}\right)$. 
Our result can be applied to e.g. the Saint-Venant system with sources terms (see e.g. [3, 10]), which is commonly used as a model for the water flow regulation in a canal with a slowly varying topography and some damping:

$$
\begin{aligned}
\partial_{t} H+\partial_{x}(H V) & =0, \\
\partial_{t} V+\partial_{x}\left(\frac{V^{2}}{2}+g H\right) & =-g \partial_{x} b-\frac{c_{f}}{2} \frac{V^{2}}{H} .
\end{aligned}
$$

In (1.8)-(1.9), $t$ is time, $x$ is the space variable, $H=H(t, x)$ is the water depth, $V=V(t, x)$ is the flow velocity in the direction parallel to the bottom, $g$ is the gravitation constant, $c_{f}$ is the friction coefficient, and $z=b(x)$ is the equation of the bottom.

Using the Riemann invariants

$$
u:=V+2 \sqrt{g H}, \quad v:=V-2 \sqrt{g H},
$$

we easily see that system (1.8)-(1.9) can be rewritten as

$$
\begin{aligned}
\partial_{t} u+\lambda(u, v) \partial_{x} u & =F(u, v), \\
\partial_{t} v-\mu(u, v) \partial_{x} v & =F(u, v)
\end{aligned}
$$

where

$$
\begin{aligned}
& \lambda:=V+\sqrt{g H}=\frac{1}{4}(3 u+v), \\
& \mu:=-V+\sqrt{g H}=-\frac{1}{4}(u+3 v), \\
& F(u, v):=-g \partial_{x} b-2 c_{f} g\left(\frac{u+v}{u-v}\right)^{2} .
\end{aligned}
$$

Thus, our results can be applied when $\left\|\partial_{x} b\right\|_{L^{\infty}(0, L)}+c_{f} \ll 1$.

The above model is valid when the function $\partial_{x} b$ takes "small values". A more accurate model, the so-called Savage-Hutter system (see [3]), reads

$$
\begin{aligned}
\partial_{t} H+\partial_{X}(H V) & =0 \\
\partial_{t} V+\partial_{X}\left(\frac{V^{2}}{2}+g \cos (\theta) H\right) & =-g \sin (\theta) .
\end{aligned}
$$

Here, $X$ denotes a curvilinear coordinate along the bottom, $\theta=\theta(X)$ is the angle of the bottom tangent with some fixed horizontal axis, $H=H(t, X)$ is the width of fluid in the normal direction at a point $X$ of the bottom, and $V(t, X)$ is the tangential velocity.

Introducing the Riemann invariants

$$
\begin{aligned}
u & :=V+2 \sqrt{g \cos (\theta) H} \\
v & :=V-2 \sqrt{g \cos (\theta) H}
\end{aligned}
$$


we derive again a system of the form (1.10)-(1.11), with $x=X$ and

$$
\begin{aligned}
\lambda & :=V+\sqrt{g \cos (\theta) H}=\frac{1}{4}(3 u+v), \\
\mu & :=-V+\sqrt{g \cos (\theta) H}=-\frac{1}{4}(u+3 v), \\
F(u, v) & :=-g \sin (\theta) .
\end{aligned}
$$

Again, our results can be applied when $|\theta| \ll 1$.

The paper is outlined as follows. The main result (Theorem 3) is stated in Section 2. Its proof is displayed in Section 3. It is divided in three parts. The first one is a rephrasing of the problem. The second part establishes the existence and uniqueness of global solutions for small initial data using Schauder's fixed-point theorem. The last one introduces some Lyapunov functions with exponential weights needed to prove the exponential convergence towards the steady state. The paper ends with an Appendix which contains the proofs of Theorem 1 and of Theorem 2 and which provides some background about linear transport equations.

\section{Stationary states And Main Result}

We are interested in the following system of balance laws

$$
\left\{\begin{array}{l}
\partial_{t} u_{\epsilon}+\lambda\left(u_{\epsilon}, v_{\epsilon}\right) \partial_{x} u_{\epsilon}=\epsilon f\left(u_{\epsilon}, v_{\epsilon}\right) \\
\partial_{t} v_{\epsilon}-\mu\left(u_{\epsilon}, v_{\epsilon}\right) \partial_{x} v_{\epsilon}=\epsilon g\left(u_{\epsilon}, v_{\epsilon}\right)
\end{array} \quad t>0, \quad x \in(0, L) .\right.
$$

System (2.1) is supplemented with the initial conditions

$$
u_{\epsilon}(0, x)=u_{0}(x), \quad v_{\epsilon}(0, x)=v_{0}(x)
$$

and the boundary conditions

$$
u_{\epsilon}(t, 0)=y_{l}(t), \quad v_{\epsilon}(t, L)=y_{r}(t) .
$$

In (2.3), the boundary data $y_{l}$ and $y_{r}$ are defined as the solutions of the initial value problem

$$
\left\{\begin{array}{l}
\frac{\mathrm{d} y_{l}}{\mathrm{~d} t}=-K \frac{y_{l}-\bar{u}}{\left|y_{l}-\bar{u}\right|^{\gamma}}, \\
\frac{\mathrm{d} y_{r}}{\mathrm{~d} t}=-K \frac{y_{r}-\bar{v}}{\left|y_{r}-\bar{v}\right|^{\gamma}}, \\
y_{l}(0)=u_{0}(0), \quad y_{r}(0)=v_{0}(1),
\end{array}\right.
$$

where $\gamma \in(0,1)$ and $K \in(0,+\infty)$ are any given numbers.

Here and in what follows, we assume that the functions $\lambda, \mu, f$ and $g$ are of class $\mathcal{C}^{2}$.

We fix a pair $(\bar{u}, \bar{v}) \in \mathbb{R}^{2}$ such that

$$
\lambda(\bar{u}, \bar{v})>0 \quad \mu(\bar{u}, \bar{v})>0,
$$

and we introduce two real numbers $c>0$ and $R>0$ such that

$$
\forall(u, v) \in \mathbb{R}^{2}, \quad\|(u, v)-(\bar{u}, \bar{v})\| \leq 2 R \Rightarrow \inf (\lambda(u, v), \mu(u, v)) \geq c .
$$

Let us construct for each $0<\epsilon \ll 1$ a pair $\left(\bar{u}_{\epsilon}, \bar{v}_{\epsilon}\right)$ of stationary states for (2.1). 
We denote by $X$ the Banach space of continuous functions from $[0, L]$ to $\mathbb{R}^{2}$ equipped with the uniform norm $\|(u, v)\|=\sup _{x \in[0, L]}\|(u(x), v(x))\|$, and by $\Omega$ the subset of $X$ consisting of the functions taking their values in the open ball $B((\bar{u}, \bar{v}), R)$; that is,

$$
\Omega=\{(u, v) \in X ;\|(u(x)-\bar{u}, v(x)-\bar{v})\|<R \quad \forall x \in[0, L]\} .
$$

We can now define a functional $F:(u, v, \epsilon) \in \Omega \times \mathbb{R} \rightarrow F(u, v, \epsilon)=(U, V) \in X$ by

$$
\begin{aligned}
U(x) & :=u(x)-\bar{u}-\epsilon \int_{0}^{x} \frac{f(u(y), v(y))}{\lambda(u(y), v(y))} d y, \\
V(x) & :=v(x)-\bar{v}-\epsilon \int_{x}^{L} \frac{g(u(y), v(y))}{\mu(u(y), v(y))} d y .
\end{aligned}
$$

It is easy to see that

(i) the set $\Omega$ is open;

(ii) $F(\bar{u}, \bar{v}, 0)=0$;

(iii) the functional $F$ is of class $\mathrm{e}^{1}$ on $\Omega$;

(iv) the differential with respect to $(u, v)$ of $F$ at $(\bar{u}, \bar{v}, 0)$ is given by $\mathrm{D}_{(u, v)} F(\bar{u}, \bar{v}, 0)=\operatorname{Id}_{X}$.

We infer from the implicit function theorem the local existence and uniqueness of a map $\epsilon \in\left[-\epsilon_{0}, \epsilon_{0}\right] \rightarrow\left(\bar{u}_{\epsilon}, \bar{v}_{\epsilon}\right) \in \Omega$ which is of class $\mathcal{C}^{1}$ and which satisfies

$$
\forall \epsilon \in\left[-\epsilon_{0}, \epsilon_{0}\right], \quad F\left(\bar{u}_{\epsilon}, \bar{v}_{\epsilon}\right)=0 .
$$

It follows then from (2.7)-(2.8) that the functions $\bar{u}_{\epsilon}$ and $\bar{v}_{\epsilon}$ are of class $\mathcal{C}^{1}$ in $[0, L]$ and that they satisfy

$$
\left\{\begin{array}{l}
\lambda\left(\bar{u}_{\epsilon}, \bar{v}_{\epsilon}\right) \partial_{x} \bar{u}_{\epsilon}=\epsilon f\left(\bar{u}_{\epsilon}, \bar{v}_{\epsilon}\right), \quad \forall x \in[0, L], \\
-\mu\left(\bar{u}_{\epsilon}, \bar{v}_{\epsilon}\right) \partial_{x} \bar{v}_{\epsilon}=\epsilon g\left(\bar{u}_{\epsilon}, \bar{v}_{\epsilon}\right), \quad \forall x \in[0, L], \\
\bar{u}_{\epsilon}(0)=\bar{u}, \\
\bar{v}_{\epsilon}(L)=\bar{v} .
\end{array}\right.
$$

We are now in a position to state the main result in this paper.

Theorem 3. There exist $\epsilon_{0}>0$ and $\delta>0$ such that for any $\epsilon \in\left[0, \epsilon_{0}\right]$, any $(\gamma, K) \in(0,1) \times$ $(0,+\infty)$, and any initial data $\left(u_{0}, v_{0}\right) \in \operatorname{Lip}([0, L])^{2}$, with

$$
\left\|u_{0}-\bar{u}\right\|_{W^{1, \infty}(0, L)} \leq \delta \quad \text { and } \quad\left\|v_{0}-\bar{v}\right\|_{W^{1, \infty}(0, L)} \leq \delta,
$$

the system (2.1)-(2.4) has a unique solution $\left(u_{\epsilon}, v_{\epsilon}\right) \in \operatorname{Lip}([0,+\infty) \times[0, L])$ satisfying (2.1) almost everywhere, and it holds

$$
\begin{aligned}
& \forall t \geq 0, \quad\left\|\left(u_{\epsilon}-\bar{u}_{\epsilon}, v_{\epsilon}-\bar{v}_{\epsilon}\right)(t)\right\|_{L^{2}(0, L)}^{2} \leq M \inf \left(1, \frac{e^{-C_{\epsilon} t}}{\epsilon^{1+\kappa}}\right)\left\|\left(u_{0}-\bar{u}_{\epsilon}, v_{0}-\bar{u}_{\epsilon}\right)\right\|_{L^{\infty}(0, L)}^{2}, \\
& \forall t \geq 0, \quad\left\|\left(u_{\epsilon}-\bar{u}_{\epsilon}, v_{\epsilon}-\bar{v}_{\epsilon}\right)(t)\right\|_{L^{\infty}(0, L)} \leq M \inf \left(1, \frac{e^{-\frac{1}{3} C_{\epsilon} t}}{\epsilon^{\frac{1+\kappa}{3}}}\right)\left\|\left(u_{0}-\bar{u}_{\epsilon}, v_{0}-\bar{u}_{\epsilon}\right)\right\|_{L^{\infty}(0, L)}^{\frac{2}{3}},
\end{aligned}
$$

where $\kappa:=\left(c \delta^{\gamma}\right) /(K L \gamma), M=M(\delta)>0$ and $C_{\epsilon}=C_{\epsilon}(\delta) \sim-\frac{c}{L} \ln (\epsilon)$ as $\epsilon \rightarrow 0^{+}$.

We shall use some Lyapunov function to prove (2.12) (see Section 3.3). 
Remark 1. We notice that for any fixed

$$
t>(1+\kappa) \frac{L}{c}=\frac{L}{c}+\frac{\delta^{\gamma}}{K \gamma},
$$

we have that

$$
\frac{e^{-C_{\epsilon} t}}{\epsilon^{1+\kappa}}=e^{\left(\ln \frac{1}{\epsilon}\right)\left[1+\kappa-t\left(\frac{c}{L}+o(1)\right)\right]}
$$

and therefore that the r.h.s. of (2.12) tends to 0 as $\epsilon \rightarrow 0^{+}$. This result, combined with the boundedness of $\left\{\left(u_{\epsilon}, v_{\epsilon}\right)\right\}_{0<\epsilon<\epsilon_{0}}$ in Lip $([0, T] \times[0, L])$ for all $T>0$, yields again the finite-time stability around $(\bar{u}, \bar{v})$ of the limit system (without source term)

$$
\begin{cases}\partial_{t} u+\lambda(u, v) \partial_{x} u=0, & t>0, x \in(0, L), \\ \partial_{t} v-\mu(u, v) \partial_{x} v=0, & t>0, x \in(0, L), \\ u(t, 0)=y_{l}(t), & t>0, \\ v(t, 1)=y_{r}(t), & t>0, \\ u(0, x)=u_{0}(x), & x \in(0, L), \\ v(0, x)=v_{0}(x) & x \in(0, L) .\end{cases}
$$

that was established in [18] with an extinction time very similar to (2.14) ( $\delta^{\gamma}$ in the r.h.s. of (2.14) being replaced by $\left.\left\|\left(u_{0}-\bar{u}, v_{0}-\bar{v}\right)\right\|_{L^{\infty}(0, L)}^{\gamma}\right)$.

\section{Proof of Theorem 3}

3.1. Reduction of the problem. We aim to show that if (2.11) holds with $\delta$ small enough, then the solutions of (2.1)-(2.4) tend to $\left(\bar{u}_{\epsilon}, \bar{v}_{\epsilon}\right)$ as $t \rightarrow+\infty$.

To this end, we introduce the functions

$$
U:=u_{\epsilon}-\bar{u}_{\epsilon}, \quad V:=v_{\epsilon}-\bar{v}_{\epsilon} .
$$

The original system (2.1) can be written

$$
\left\{\begin{aligned}
\partial_{t} U+\lambda\left(\bar{u}_{\epsilon}+U, \bar{v}_{\epsilon}+V\right) \partial_{x} U=\epsilon\left[f\left(\bar{u}_{\epsilon}+U, \bar{v}_{\epsilon}+V\right)-f\left(\bar{u}_{\epsilon}, \bar{v}_{\epsilon}\right)\right] & \\
& -\partial_{x} \bar{u}_{\epsilon}\left[\lambda\left(\bar{u}_{\epsilon}+U, \bar{v}_{\epsilon}+V\right)-\lambda\left(\bar{u}_{\epsilon}, \bar{v}_{\epsilon}\right)\right], \\
\partial_{t} V-\mu\left(\bar{u}_{\epsilon}+\right. & \left.U, \bar{v}_{\epsilon}+V\right) \partial_{x} V=\epsilon\left[g\left(\bar{u}_{\epsilon}+U, \bar{v}_{\epsilon}+V\right)-g\left(\bar{u}_{\epsilon}, \bar{v}_{\epsilon}\right)\right] \\
& +\partial_{x} \bar{v}_{\epsilon}\left[\mu\left(\bar{u}_{\epsilon}+U, \bar{v}_{\epsilon}+V\right)-\mu\left(\bar{u}_{\epsilon}, \bar{v}_{\epsilon}\right)\right] .
\end{aligned}\right.
$$

The boundary conditions become

$$
\left\{\begin{array}{l}
U(t, 0)=y_{l}(t)-\bar{u} \\
V(t, L)=y_{r}(t)-\bar{v}
\end{array}\right.
$$

where $y_{l}$ and $y_{r}$ still solve (2.4), and the initial condition read

$$
U(0, x)=U_{0}(x):=u_{0}(x)-\bar{u}_{\epsilon}(x), \quad V(0, x)=V_{0}(x):=v_{0}(x)-\bar{v}_{\epsilon}(x) .
$$

Note that $y_{l}(t)=\bar{u}\left(\operatorname{resp} . y_{r}(t)=\bar{v}\right)$ for $t \geq(\gamma K)^{-1}\left|U_{0}(0)\right|^{\gamma}\left(\operatorname{resp} . t \geq(\gamma K)^{-1}\left|V_{0}(1)\right|^{\gamma}\right)$. Therefore, if $\left\|U_{0}\right\|_{L^{\infty}(0, L)} \leq \delta$ and $\left\|V_{0}\right\|_{L^{\infty}(0, L)} \leq \delta$, then we have

$$
U(t, 0)=V(t, 1)=0 \quad \forall t \geq(\gamma K)^{-1} \delta^{\gamma} .
$$


Thanks to the definition of $\Omega$, we notice that for any $\epsilon \in\left[-\epsilon_{0}, \epsilon_{0}\right]$, it holds

$$
\forall x \in[0, L], \quad\left\|\left(\bar{u}_{\epsilon}(x), \bar{v}_{\epsilon}(x)\right)-(\bar{u}, \bar{v})\right\|<R .
$$

For any given $r>0$ and any $f: \mathbb{R}^{2} \rightarrow \mathbb{R}$, let

$$
\|f\|_{r}:=\sup \{|f(u, v)| ; \quad(u, v) \in B((\bar{u}, \bar{v}) ; r)\} .
$$

In the following, for any function $f=f(u, v)$, the quantity $\|f\|$ will always denote $\|f\|_{R}$ where $R$ is as in (2.6). (Note that $R$ does not depend on $\epsilon$.) For $h:[0, L] \rightarrow \mathbb{R}$, we denote $\|h\|_{[0, L]}=\sup \{|h(x)| ; x \in[0, L]\}$.

Using (2.10), we see that

$$
\begin{aligned}
&\left\|\partial_{x} \bar{u}_{\epsilon}\right\|_{[0, L]} \leq \epsilon \frac{\|f\|}{c}, \\
&\left\|\partial_{x} \bar{v}_{\epsilon}\right\|_{[0, L]} \leq \epsilon \frac{\|g\|}{c} .
\end{aligned}
$$

Differentiating in (2.10), we infer that

$$
\begin{aligned}
& \left\|\partial_{x x}^{2} \bar{u}_{\epsilon}\right\|_{[0, L]} \leq \epsilon^{2}\left(\frac{\|f\| \cdot\left\|\partial_{1} f\right\|+\|g\| \cdot\left\|\partial_{2} f\right\|}{c^{2}}+\frac{\left\|\partial_{1} \lambda\right\| \cdot\|f\|^{2}}{c^{3}}+\frac{\left\|\partial_{2} \lambda\right\| \cdot\|f\| \cdot\|g\|}{c^{3}}\right), \\
& \left\|\partial_{x x}^{2} \bar{v}_{\epsilon}\right\|_{[0, L]} \leq \epsilon^{2}\left(\frac{\|f\| \cdot\left\|\partial_{1} g\right\|+\|g\| \cdot\left\|\partial_{2} g\right\|}{c^{2}}+\frac{\left\|\partial_{2} \mu\right\| \cdot\|g\|^{2}}{c^{3}}+\frac{\left\|\partial_{1} \mu\right\| \cdot\|f\| \cdot\|g\|}{c^{3}}\right) .
\end{aligned}
$$

We now simplify the notations by setting

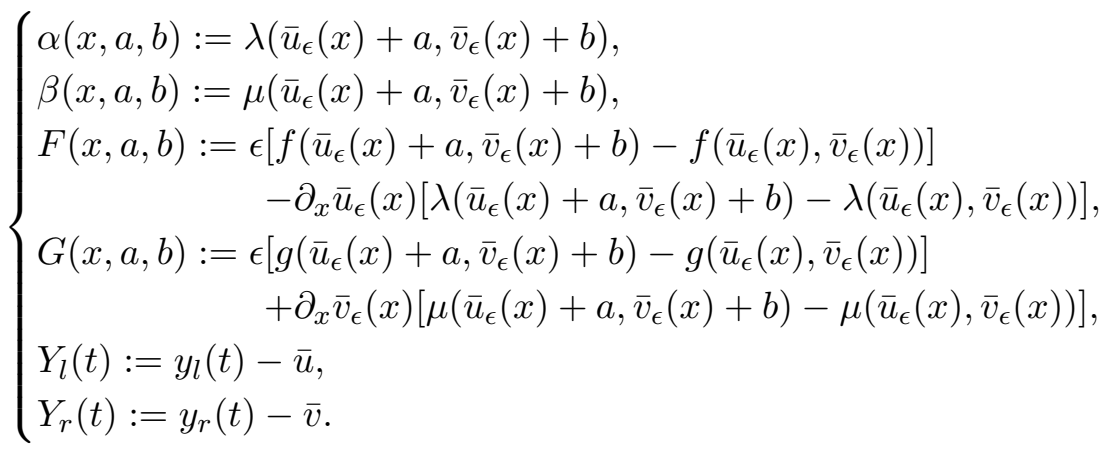

The system (3.1) can be written as

$$
\left\{\begin{array}{l}
\partial_{t} U+\alpha(x, U, V) \partial_{x} U=F(x, U, V) \\
\partial_{t} V-\beta(x, U, V) \partial_{x} V=G(x, U, V)
\end{array}\right.
$$

It is supplemented with the initial conditions

$$
U(0, x)=U_{0}(x), \quad V(0, x)=V_{0}(x),
$$

and the boundary conditions

$$
\left\{\begin{array}{l}
U(t, 0)=Y_{l}(t), \\
V(t, L)=Y_{r}(t),
\end{array}\right.
$$


where the functions $Y_{l}$ and $Y_{r}$ solve the system

$$
\left\{\begin{array}{l}
\frac{\mathrm{d} Y_{l}}{\mathrm{~d} t}=-K \frac{Y_{l}}{\left|Y_{l}\right|^{\gamma}}, \\
\frac{\mathrm{d} Y_{r}}{\mathrm{~d} t}=-K \frac{Y_{r}}{\left|Y_{r}\right|^{\gamma}}, \\
Y_{l}(0)=u_{0}(0)-\bar{u}, \quad Y_{r}(0)=v_{0}(1)-\bar{v} .
\end{array}\right.
$$

The functions $\alpha, \beta, F$ and $G$ enjoy the following properties:

$$
\begin{aligned}
\forall(a, b) \in B((0,0), R), & \inf (\alpha(x, a, b), \beta(x, a, b)) \geq c ; \\
\forall x \in[0, L], & F(x, 0,0)=0=G(x, 0,0) ;
\end{aligned}
$$

$\forall x \in[0, L], \forall(a, b) \in B((0,0), R)$

$$
\begin{aligned}
& \frac{|F(x, a, b)|}{\|(a, b)\|} \leq \epsilon\left(\|D f\|_{B((\bar{u}, \bar{v}), 2 R)}+\frac{\|f\|_{B((\bar{u}, \bar{v}), R)}}{c}\|D \lambda\|_{B((\bar{u}, \bar{v}), 2 R)}\right) ; \\
& \frac{|G(x, a, b)|}{\|(a, b)\|} \leq \epsilon\left(\|D g\|_{B((\bar{u}, \bar{v}), 2 R)}+\frac{\|g\|_{B((\bar{u}, \bar{v}), R)}}{c}\|D \mu\|_{B((\bar{u}, \bar{v}), 2 R)}\right)
\end{aligned}
$$

$$
\begin{aligned}
& \forall x \in[0, L], \quad \forall(a, b) \in B((0,0), R) \\
& \left\{\begin{array}{l}
\left|\partial_{x} F(x, a, b)\right| \leq \frac{2 \epsilon^{2}}{c^{2}}\left(1+\frac{\|\lambda\|}{c}\right)\left(c\|f\| \cdot\left\|\partial_{1} f\right\|+c\|g\| \cdot\left\|\partial_{2} f\right\|+\left\|\partial_{1} \lambda\right\| \cdot\|f\|^{2}+\left\|\partial_{2} \lambda\right\| \cdot\|f\| .\|g\|\right) \\
\left|\partial_{x} G(x, a, b)\right| \leq \frac{2 \epsilon^{2}}{c^{2}}\left(1+\frac{\|\mu\| \mid}{c}\right)\left(c\left\|\partial_{1} g\right\| \cdot\|f\|+c\left\|\partial_{2} g\right\| \cdot\|g\|+\|g\|^{2} \cdot\left\|\partial_{2} \mu\right\|+\|g\| \cdot\|f\| \cdot\left\|\partial_{1} \mu\right\|\right) \\
\left|\partial_{a} F(x, a, b)\right| \leq \epsilon\left(\left\|\partial_{1} f\right\|_{2 R}+\frac{\left.\left\|\partial_{1} \lambda\right\|\right|_{2 R} \cdot\|f\|}{c}\right) \\
\left|\partial_{b} F(x, a, b)\right| \leq \epsilon\left(\left\|\partial_{2} f\right\|_{2 R}+\frac{\left\|\partial_{2} \lambda\right\|\left\|_{2 R} \cdot\right\| f \|}{c}\right) \\
\left|\partial_{a} G(x, a, b)\right| \leq \epsilon\left(\left\|\partial_{1} g\right\|_{2 R}+\frac{\left\|\partial_{1} \mu\right\|_{2 R} \cdot\|g\|}{c}\right) \\
\left|\partial_{b} G(x, a, b)\right| \leq \epsilon\left(\left\|\partial_{2} g\right\|_{2 R}+\frac{\left\|\partial_{2} \mu\right\|_{2 R} \cdot\|g\|}{c}\right)
\end{array}\right.
\end{aligned}
$$

3.2. The fixed-point argument. We show in this section the existence and uniqueness of the solution of (3.1)-(3.3) for $\epsilon$ small enough. Introduce some positive constants $I_{0}, I_{1}, P$, and $M$ such that

$$
\begin{gathered}
\left\|U_{0}\right\|_{L^{\infty}(0, L)} \leq I_{0}, \quad\left\|V_{0}\right\|_{L^{\infty}(0, L)} \leq I_{0}, \\
\left\|U_{0}^{\prime}\right\|_{L^{\infty}(0, L)} \leq I_{1}, \quad\left\|V_{0}^{\prime}\right\|_{L^{\infty}(0, L)} \leq I_{1}, \\
\sup \left(\left\|\partial_{x} F\right\|_{\infty},\left\|\partial_{a} F\right\|_{\infty},\left\|\partial_{b} F\right\|_{\infty},\left\|\partial_{x} G\right\|_{\infty},\left\|\partial_{a} G\right\|_{\infty},\left\|\partial_{b} G\right\|_{\infty}\right) \leq P,
\end{gathered}
$$

and

$$
\sup \left(\|\alpha\|_{\infty},\left\|\partial_{x} \alpha\right\|_{\infty},\left\|\partial_{a} \alpha\right\|_{\infty},\left\|\partial_{b} \alpha\right\|_{\infty},\|\beta\|_{\infty},\left\|\partial_{x} \beta\right\|_{\infty},\left\|\partial_{a} \beta\right\|_{\infty},\left\|\partial_{b} \beta\right\|_{\infty}\right) \leq M .
$$

We notice that we can assume that 
(i) the constants $I_{0}, I_{1}$ are small by picking the numbers $\delta$ and $\epsilon_{0}$ in Theorem 3 small enough;

(ii) the constant $P$ is small by adjusting $\epsilon$ thanks to (3.12);

(iii) the constant $M$ is bounded for $\epsilon \in\left[0, \epsilon_{0}\right]$.

To display our fixed-point argument based on Schauder fixed-point theorem, we have to define (i) a class of functions containing the desired solution; (ii) a map whose fixed-point is the desired solution of (3.1)-(3.3). This is done in the following two definitions.

Definition 1. Given two positive constants $A$ and $B$, we define $\mathcal{D}$ as the set of functions $(U, V) \in \operatorname{Lip}\left(\mathbb{R}^{+} \times[0, L]\right)^{2}$ such that

$$
\begin{gathered}
\forall(t, x) \in \mathbb{R}^{+} \times[0, L], \quad\left\{\begin{array}{l}
|U(t, x)| \leq A, \\
|V(t, x)| \leq A ;
\end{array}\right. \\
\forall(t, x, y) \in \mathbb{R}^{+} \times[0, L]^{2}, \quad\left\{\begin{array}{l}
|U(t, x)-U(t, y)| \leq B|x-y|, \\
|V(t, x)-V(t, y)| \leq B|x-y| ;
\end{array}\right.
\end{gathered}
$$

and

$$
\forall(t, s, y) \in \mathbb{R}^{+} \times \mathbb{R}^{+} \times[0, L], \quad\left\{\begin{array}{l}
|U(t, x)-U(s, x)| \leq B|t-s|, \\
|V(t, x)-V(s, x)| \leq B|t-s| .
\end{array}\right.
$$

Definition 2. Given $(U, V) \in \mathcal{D}$, we introduce the unique solution $(\tilde{U}, \tilde{V})$ (thanks to Theorem 7 in Appendix) of the system

$$
\left\{\begin{array}{l}
\partial_{t} \tilde{U}+\alpha(x, U, V) \partial_{x} \tilde{U}=F(x, U, V), \\
\partial_{t} \tilde{V}-\beta(x, U, V) \partial_{x} \tilde{V}=G(x, U, V), \\
\tilde{U}(t, 0)=Y_{l}(t), \\
\tilde{V}(t, L)=Y_{r}(t), \\
\tilde{U}(0, x)=U_{0}(x), \\
\tilde{V}(0, x)=V_{0}(x) .
\end{array}\right.
$$

We set $\mathcal{F}(U, V):=(\tilde{U}, \tilde{V})$.

The following result is classical (see e.g. [?]).

Proposition 1. The function $d:\left[\mathfrak{C}^{0}\left(\left[0,+\infty[\times[0, L])^{2}\right]^{2} \rightarrow \mathbb{R}^{+}\right.\right.$defined by

$$
d((U, V),(u, v)):=\sum_{n=1}^{+\infty} \frac{1}{2^{n}} \frac{\|U-u\|_{L^{\infty}([0, n] \times[0, L])}+\|V-v\|_{L^{\infty}([0, n] \times[0, L])}}{1+\|U-u\|_{L^{\infty}([0, n] \times[0, L])}+\|V-v\|_{L^{\infty}([0, n] \times[0, L])}}
$$

is a distance and $\left[\mathrm{C}^{0}\left([0,+\infty[\times[0, L])]^{2}\right.\right.$ endowed with this distance is a Fréchet Space. On the other hand, $\mathcal{D}$ is a convex compact subset of $\left[\mathfrak{C}^{0}\left([0,+\infty[\times[0, L])]^{2}\right.\right.$.

We first have to show that $\mathcal{F}$ maps $\mathcal{D}$ into itself for an appropriate choice of the constants.

Proposition 2. For any $A, B, c, K, L, M$ and $\gamma$ in $(0,+\infty)$, there exist some numbers $I_{0}, I_{1}$ and $P$ in $(0,+\infty)$ such that $\mathcal{F}(\mathcal{D}) \subset \mathcal{D}$. 
Proof. Using Corollary 1 in Appendix, we obtain the following estimates:

$$
\begin{gathered}
\|\tilde{U}\|_{L^{\infty}} \leq \frac{2 L}{c} P A+I_{0} \\
\|\tilde{V}\|_{L^{\infty}} \leq \frac{2 L}{c} P A+I_{0} \\
\left\|\partial_{x} \tilde{U}\right\|_{L^{\infty}} \leq \frac{L}{c} \exp \left(\frac{L}{c} M(1+2 B)\right)\left(P(1+2 B)+\frac{\sup \left(2 P A+K I_{0}^{1-\gamma}, c I_{1}\right)}{L}\right) ; \\
\left\|\partial_{x} \tilde{V}\right\|_{L^{\infty}} \leq \frac{L}{c} \exp \left(\frac{L}{c} M(1+2 B)\right)\left(P(1+2 B)+\frac{\sup \left(2 P A+K I_{0}^{1-\gamma}, c I_{1}\right)}{L}\right) \\
\left\|\partial_{t} \tilde{U}\right\|_{L^{\infty}} \leq \frac{M L}{c} \exp \left(\frac{L}{c} M(1+2 B)\right)\left(P(1+2 B)+\frac{\sup \left(2 P A+K I_{0}^{1-\gamma}, c I_{1}\right)}{L}\right)+2 P A \\
\left\|\partial_{t} \tilde{V}\right\|_{L^{\infty}} \leq \frac{M L}{c} \exp \left(\frac{L}{c} M(1+2 B)\right)\left(P(1+2 B)+\frac{\sup \left(2 P A+K I_{0}^{1-\gamma}, c I_{1}\right)}{L}\right)+2 P A .
\end{gathered}
$$

It is thus sufficient to show that for $I_{0}$ and $I_{1}$ small enough, one can choose $P$ sufficiently small so that there exist some numbers $A>0$ and $B>0$ with

$$
\begin{aligned}
\frac{2 L}{c} P A+I_{0} & \leq A, \\
\frac{L}{c} \exp \left(\frac{L}{c} M(1+2 B)\right)\left(P(1+2 B)+\frac{\sup \left(2 P A+K I_{0}^{1-\gamma}, c I_{1}\right)}{L}\right) & \leq B, \\
\frac{M L}{c} \exp \left(\frac{L}{c} M(1+2 B)\right)\left(P(1+2 B)+\frac{\sup \left(2 P A+K I_{0}^{1-\gamma}, c I_{1}\right)}{L}\right)+2 P A & \leq B .
\end{aligned}
$$

For given $A, B, M, I_{0}$ and $I_{1}$ in $(0,+\infty)$, it is thus sufficient to impose that as $P \rightarrow 0^{+}$, the limit of the leftsided terms in (3.23)-(3.25) are strictly less than those of the corresponding rightsided terms; that is,

$$
\begin{aligned}
I_{0} & <A, \\
\frac{L}{c} \exp \left(\frac{L}{c} M(1+2 B)\right) \frac{\sup \left(K I_{0}^{1-\gamma}, c I_{1}\right)}{L} & <B, \\
\frac{M L}{c} \exp \left(\frac{L}{c} M(1+2 B)\right) \frac{\sup \left(K I_{0}^{1-\gamma}, c I_{1}\right)}{L} & <B .
\end{aligned}
$$

Now, it is clear that given $A, B, c, K, L, M$ and $\gamma$ in $(0,+\infty)$, the conditions (3.26)-(3.28) are fulfilled by choosing $I_{0}$ and $I_{1}$ small enough.

Next, we have to show the continuity of the map $\mathcal{F}$.

Proposition 3. The map $\mathcal{F}: \mathcal{D} \rightarrow \mathcal{D}$ is continuous. 
Proof. Let $\left\{\left(U_{n}, V_{n}\right)\right\} \subset \mathcal{D}$ be any sequence such that

$$
d\left(\left(U_{n}, V_{n}\right),(U, V)\right) \underset{n \rightarrow+\infty}{\rightarrow} 0
$$

for some $(U, V) \in \mathcal{D}$. This is equivalent to saying that $\left(U_{n}\right)_{n \geq 0}$ tends to $U$ (and $\left(V_{n}\right)_{n \geq 0}$ tends to $V)$ uniformly on all the sets $[0, T] \times[0, L], T>0$. This yields that $\alpha\left(., U_{n}, V_{n}\right) \rightarrow \alpha(., U, V)$ and $\beta\left(., U_{n}, V_{n}\right) \rightarrow \beta(., U, V)$ uniformly on all the sets $[0, T] \times[0, L], T>0$. Using Theorem 6 in Appendix, we deduce that $\mathcal{F}\left(U_{n}, V_{n}\right)$ converges uniformly on all the sets $[0, T] \times[0, L], T>0$, to a pair $(\tilde{U}, \tilde{V}) \in \mathcal{D}$ which is the unique solution of the system

$$
\left\{\begin{array}{l}
\partial_{t} \tilde{U}+\alpha(x, U, V) \partial_{x} \tilde{U}=F(x, U, V), \\
\partial_{t} \tilde{V}-\beta(x, U, V) \partial_{x} \tilde{V}=G(x, U, V), \\
\tilde{U}(t, 0)=Y_{l}(t), \\
\tilde{V}(t, L)=Y_{r}(t), \\
\tilde{U}(0, x)=U_{0}(x), \\
\tilde{V}(0, x)=V_{0}(x) .
\end{array}\right.
$$

By Definition 2, we conclude that $(\tilde{U}, \tilde{V})=\mathcal{F}(U, V)$. It follows that $\mathcal{F}$ is continuous.

We are now in a position to prove that $\mathcal{F}$ has a fixed-point in $\mathcal{D}$.

Proposition 4. The map $\mathcal{F}$ has a fixed-point in $\mathcal{D}$, and therefore the system (2.1)-(2.3) has at least one solution.

Proof. As the map $\mathcal{F}: \mathcal{D} \rightarrow \mathcal{D}$ is continuous and $\mathcal{D}$ is a convex, compact subset of the Fréchet space $\left[\mathcal{C}^{0}\left([0,+\infty[\times[0, L])]^{2}\right.\right.$, it has at least one fixed-point by Tihonov fixed-point theorem (see e.g. [20, Corollary 9.6].) The pair $(U, V)$ solves (3.6)-(3.8), while the pair $\left(u_{\epsilon}, v_{\epsilon}\right):=\left(U+\bar{u}_{\epsilon}, V+\right.$ $\left.\bar{v}_{\epsilon}\right)$ solves $(2.1)-(2.3)$.

We are now concerned with the uniqueness of the solution of the system (2.1)-(2.3).

Proposition 5. There is at most one solution to (2.1)-(2.3) in the class Lip $([0, T] \times[0, L])^{2}$.

Proof. Clearly, it is sufficient to prove the uniqueness of the solution $(U, V)$ to the system (3.6)(3.8).

Assume given $U_{0}, V_{0} \in \operatorname{Lip}([0, L])$ and two pairs $\left(U^{i}, V^{i}\right) \in \operatorname{Lip}([0, T] \times[0, L])^{2}(i=1,2)$ of solutions of (3.6)-(3.8); that is, we have for $i=1,2$

$$
\left\{\begin{array}{l}
\partial_{t} U^{i}+\alpha\left(x, U^{i}, V^{i}\right) \partial_{x} U^{i}=F\left(x, U^{i}, V^{i}\right) \\
\partial_{t} V^{i}-\beta\left(x, U^{i}, V^{i}\right) \partial_{x} V^{i}=G\left(x, U^{i}, V^{i}\right) \\
U^{i}(t, 0)=Y_{l}(t), \quad V^{i}(t, L)=Y_{r}(t) \\
U^{i}(0, x)=U_{0}(x), \quad V^{i}(0, x)=V_{0}(x) .
\end{array}\right.
$$

Set $\hat{U}:=U^{1}-U^{2}, \hat{V}:=V^{1}-V^{2}$, and

$$
\begin{aligned}
\hat{\alpha} & :=\alpha\left(x, U^{1}, V^{1}\right)-\alpha\left(x, U^{2}, V^{2}\right), \\
\hat{\beta} & :=\beta\left(x, U^{1}, V^{1}\right)-\beta\left(x, U^{2}, V^{2}\right), \\
\hat{F} & :=F\left(x, U^{1}, V^{1}\right)-F\left(x, U^{2}, V^{2}\right), \\
\hat{G} & :=G\left(x, U^{1}, V^{1}\right)-G\left(x, U^{2}, V^{2}\right) .
\end{aligned}
$$


Then we see that $\hat{U}, \hat{V} \in \operatorname{Lip}([0, T] \times[0, L])$ and that the pair $(\hat{U}, \hat{V})$ solves the system

$$
\begin{aligned}
\partial_{t} \hat{U}+\alpha\left(x, U^{1}, V^{1}\right) \partial_{x} \hat{U}+\hat{\alpha} \partial_{x} U^{2} & =\hat{F}, \\
\partial_{t} \hat{V}-\beta\left(x, U^{1}, V^{1}\right) \partial_{x} \hat{V}-\hat{\beta} \partial_{x} V^{2} & =\hat{G}, \\
\hat{U}(t, 0)=\hat{V}(t, L) & =0, \\
\hat{U}(0, x)=\hat{V}(0, x) & =0 .
\end{aligned}
$$

After multiplying (3.30) (resp. (3.31)) by $\hat{U}$ (resp. by $\hat{V}$ ), and integrating over $(0, t) \times(0, L)$, we obtain

$$
\begin{aligned}
\frac{1}{2}\left(\|\hat{U}\|_{L^{2}(0, L)}^{2}+\|\hat{V}\|_{L^{2}(0, L)}^{2}\right)= & \int_{0}^{t} \int_{0}^{L}[\hat{F} \hat{U}+\hat{G} \hat{V}] d x d \tau \\
& -\int_{0}^{t} \int_{0}^{L}\left[\alpha\left(x, U^{1}, V^{1}\right) \hat{U} \partial_{x} \hat{U}-\beta\left(x, U^{1}, V^{1}\right) \hat{V} \partial_{x} \hat{V}\right] d x d \tau \\
& -\int_{0}^{t} \int_{0}^{L}\left[\hat{\alpha} \hat{U} \partial_{x} U^{2}-\hat{\beta} \hat{V} \partial_{x} V^{2}\right] d x d \tau=: I_{1}-I_{2}-I_{3} .
\end{aligned}
$$

Then

$$
\left|I_{1}\right| \leq \int_{0}^{t} \int_{0}^{L}[P(|\hat{U}|+|\hat{V}|)|\hat{U}|+P(|\hat{U}|+|\hat{V}|)|\hat{V}|] d x d \tau \leq 2 P \int_{0}^{t} \int_{0}^{L}\left(|\hat{U}|^{2}+|\hat{V}|^{2}\right) d x d \tau .
$$

Similarly,

$$
\left|I_{3}\right| \leq M B \int_{0}^{t} \int_{0}^{L}(|\hat{U}|+|\hat{V}|)^{2} d x d \tau \leq 2 M B \int_{0}^{t} \int_{0}^{L}\left(|\hat{U}|^{2}+|\hat{V}|^{2}\right) d x d \tau .
$$

On the other hand, integrating by part in $I_{2}$ yields

$$
\begin{aligned}
-I_{2}= & \int_{0}^{t} \int_{0}^{L}\left(\partial_{x}\left[\alpha\left(x, U^{1}, V^{1}\right)\right]|\hat{U}|^{2}-\partial_{x}\left[\beta\left(x, U^{1}, V^{1}\right)\right]|\hat{V}|^{2}\right) d x d \tau \\
& -\int_{0}^{t}\left[\alpha\left(x, U^{1}, V^{1}\right)|\hat{U}|^{2}-\beta\left(x, U^{1}, V^{1}\right)|\hat{V}|^{2}\right]_{0}^{L} d \tau \\
\leq & \int_{0}^{t} \int_{0}^{L}\left(\partial_{x}\left[\alpha\left(x, U^{1}, V^{1}\right)\right]|\hat{U}|^{2}-\partial_{x}\left[\beta\left(x, U^{1}, V^{1}\right)\right]|\hat{V}|^{2}\right) d x d \tau \\
\leq & M(1+2 B) \int_{0}^{t} \int_{0}^{L}\left(|\hat{U}|^{2}+|\hat{V}|^{2}\right) d x d \tau
\end{aligned}
$$

where we used the definitions of $\alpha, \beta, M, B,(2.6)$ and (3.32). Gathering together all the estimates, we arrive to

$$
\int_{0}^{L}\left[|\hat{U}(t, x)|^{2}+|\hat{V}(t, x)|^{2}\right] d x \leq[M(1+4 B)+2 P] \int_{0}^{t} \int_{0}^{L}\left(|\hat{U}|^{2}+|\hat{V}|^{2}\right) d x d \tau, \quad \forall t \geq 0 .
$$

An application of Gronwall's lemma yields $\hat{U} \equiv 0$ and $\hat{V} \equiv 0$. 
3.3. Lyapunov Functions and Decay Rates. Let $\left(U, V, Y_{r}, Y_{l}\right)$ denote the solution of system (3.6)-(3.9).

To simplify the computations, we denote by $\tilde{C}$ a positive constant such that for all $x \in[0, L]$ and all $(a, b) \in B((0,0), R)$, we have

$$
|F(x, a, b)| \leq \frac{\tilde{C} \epsilon}{2}(|a|+|b|), \quad|G(x, a, b)| \leq \frac{\tilde{C} \epsilon}{2}(|a|+|b|)
$$

and such that for all $(t, x) \in \mathbb{R}^{+} \times[0, L]$, we have

$$
\sup (\alpha(x, U(t, x), V(t, x)), \beta(x, U(t, x), V(t, x))) \leq \tilde{C}
$$

and

$$
\left|\frac{d}{d x}(\alpha(x, U(t, x), V(t, x)))\right| \leq \tilde{C}, \quad\left|\frac{d}{d x}(\beta(x, U(t, x), V(t, x)))\right| \leq \tilde{C} .
$$

We first introduce a Lyapunov function to investigate the long-time behavior of $(U, V)$.

Definition 3. Given any $\theta>0$, let the function $L_{\theta}$ be defined by

$$
\mathcal{L}_{\theta}(a, b):=\int_{0}^{L}\left[a^{2}(x) e^{-\theta x}+b^{2}(x) e^{-\theta(L-x)}\right] d x \quad \forall(a, b) \in\left[L^{2}(0, L)\right]^{2} .
$$

Then we have the following result.

Proposition 6. For almost every $t \in \mathbb{R}^{+}$, it holds

$$
\frac{d}{d t} \mathcal{L}_{\theta}(U(t, .), V(t, .)) \leq\left(\tilde{C} \epsilon \frac{3+e^{\theta L}}{2}+\tilde{C}-c \theta\right) \mathcal{L}_{\theta}(U(t, .), V(t, .))+\tilde{C}\left(Y_{r}^{2}(t)+Y_{l}^{2}(t)\right) .
$$

Proof. Since $U$ and $V$ are uniformly bounded and Lipschitz continuous, and hence differentiable almost everywhere by Rademacher theorem, the derivative of $\mathcal{L}_{\theta}(U(t,),. V(t,)$.$) exists almost$ everywhere and it is obtained by differentiating the integrand with respect to $t$. Thus we obtain

$$
\begin{aligned}
\frac{d}{d t} \mathcal{L}_{\theta}(U(t, .), V(t, .))= & \int_{0}^{L} 2 F(x, U(t, x), V(t, x)) U(t, x) e^{-\theta x} d x \\
& +\int_{0}^{L} 2 G(x, U(t, x), V(t, x)) V(t, x) e^{-\theta(L-x)} d x \\
& -\int_{0}^{L} \alpha(x, U(t, x), V(t, x)) \partial_{x}\left(U^{2}\right)(t, x) e^{-\theta x} d x \\
& +\int_{0}^{L} \beta(x, U(t, x), V(t, x)) \partial_{x}\left(V^{2}\right)(t, x) e^{-\theta(L-x)} d x \\
= & : I_{1}+I_{2}+I_{3}+I_{4} .
\end{aligned}
$$

Let us begin with $I_{3}$.

$$
\begin{aligned}
I_{3}=-\left[e^{-\theta x} \alpha(x, U(t, x), V(t, x)) U^{2}(t, x)\right]_{0}^{L}- & \theta \int_{0}^{L} \alpha(x, U(t, x), V(t, x)) U^{2}(t, x) e^{-\theta x} d x \\
& +\int_{0}^{L} \frac{d}{d x}(\alpha(x, U(t, x), V(t, x))) U^{2}(t, x) e^{-\theta x} d x .
\end{aligned}
$$


As far $I_{4}$ is concerned, we have that

$$
\begin{array}{r}
I_{4}=\left[e^{-\theta(L-x)} \beta(x, U(t, x), V(t, x)) V^{2}(t, x)\right]_{0}^{L}-\theta \int_{0}^{L} \beta(x, U(t, x), V(t, x)) V^{2}(t, x) e^{-\theta(L-x)} d x \\
-\int_{0}^{\mathcal{L}} \frac{d}{d x}(\beta(x, U(t, x), V(t, x))) V^{2}(t, x) e^{-\theta(L-x)} d x .
\end{array}
$$

Using (3.10) and (3.35), we infer that

$$
\begin{aligned}
I_{3}+I_{4} \leq \quad \alpha(0, U(t, 0), V(t, 0)) U^{2}(t, 0)+\beta(L, U(t, L), V(t, L)) V^{2}(t, L) \\
\\
+(\tilde{C}-\theta c) \mathcal{L}_{\theta}(U(t, .), V(t, .)) .
\end{aligned}
$$

Let us evaluate the remaining terms $I_{1}$ and $I_{2}$. We have that

$$
\begin{aligned}
I_{1}+I_{2} & \leq \tilde{C} \epsilon \int_{0}^{L}\left[U^{2}(t, x) e^{-\theta x}+V^{2}(t, x) e^{-\theta(L-x)}+U(t, x) V(t, x)\left(e^{-\theta(L-x)}+e^{-\theta x}\right)\right] d x \\
& \leq \tilde{C} \epsilon \mathcal{L}_{\theta}(U(t, .), V(t, .))+\tilde{C} \epsilon \int_{0}^{L} \frac{U^{2}(t, x)+V^{2}(t, x)}{2}\left(e^{-\theta(L-x)}+e^{-\theta x}\right) d x \\
& \leq \tilde{C} \epsilon \frac{3+e^{\theta L}}{2} \mathcal{L}_{\theta}(U(t, .), V(t, .)),
\end{aligned}
$$

where we used twice in the last inequality the following estimate

$$
\forall x \in[0, L], \quad e^{-\theta(L-x)}+e^{-\theta x} \leq\left(e^{\theta L}+1\right) e^{-\theta x} .
$$

The proof of Proposition 6 is completed by gathering together (3.36) and (3.37).

Next, we introduce another Lyapunov function for the dynamics of the boundary conditions.

Definition 4. Given any $\theta>0$, let the function $\tilde{\mathcal{L}}_{\theta}$ be defined by

$$
\forall(a, b) \in \mathbb{R}^{2}, \quad \tilde{\mathcal{L}}_{\theta}(a, b):=\frac{\tilde{C}|a|^{\gamma+2}}{K(\gamma+2)} e^{\theta \frac{c}{K \gamma}|a|^{\gamma}}+\frac{\tilde{C}|b|^{\gamma+2}}{K(\gamma+2)} e^{\theta \frac{c}{K \gamma}|b|^{\gamma}} .
$$

Then the following result holds.

Proposition 7. We have for all $t \geq 0$

$$
\frac{d}{d t} \tilde{\mathcal{L}}_{\theta}\left(Y_{l}(t), Y_{r}(t)\right) \leq-c \theta \tilde{\mathcal{L}}_{\theta}\left(Y_{l}(t), Y_{r}(t)\right)-\tilde{C}\left(Y_{l}^{2}(t)+Y_{r}^{2}(t)\right) .
$$

Proof. From

$$
\begin{aligned}
& \partial_{a} \tilde{\mathcal{L}}_{\theta}(a, b)=\frac{\tilde{C}}{K(\gamma+2)}\left((\gamma+2) a|a|^{\gamma}+\frac{\theta c}{K} a|a|^{2 \gamma}\right) e^{\theta \frac{c}{K \gamma}|a|^{\gamma}}, \\
& \partial_{b} \tilde{\mathcal{L}}_{\theta}(a, b)=\frac{\tilde{C}}{K(\gamma+2)}\left((\gamma+2) b|b|^{\gamma}+\frac{\theta c}{K} b|b|^{2 \gamma}\right) e^{\theta \frac{c}{K \gamma}|b|^{\gamma}},
\end{aligned}
$$

and

$$
\begin{aligned}
& \dot{Y}_{g}(t)=-K \frac{Y_{l}(t)}{\left|Y_{l}(t)\right|^{\gamma}}, \\
& \dot{Y}_{d}(t)=-K \frac{Y_{r}(t)}{\left|Y_{r}(t)\right|^{\gamma}},
\end{aligned}
$$


we infer that

$$
\begin{aligned}
\frac{d}{d t} \tilde{\mathcal{L}}_{\theta}\left(Y_{l}(t), Y_{r}(t)\right) & =\dot{Y}_{g}(t) \partial_{a} \tilde{\mathcal{L}}_{\theta}\left(Y_{l}(t), Y_{r}(t)\right)+\dot{Y}_{d}(t) \partial_{b} \tilde{\mathcal{L}}_{\theta}\left(Y_{l}(t), Y_{r}(t)\right) \\
& =-\tilde{C} Y_{l}^{2}(t) e^{\theta \frac{c}{K \gamma}\left|Y_{l}(t)\right|^{\gamma}}-\tilde{C} Y_{r}^{2}(t) e^{\theta \frac{c}{K \gamma}\left|Y_{r}(t)\right|^{\gamma}}-c \theta \tilde{\mathcal{L}}_{\theta}\left(Y_{l}(t), Y_{r}(t)\right) \\
& \leq-\tilde{C} Y_{l}^{2}(t)-\tilde{C} Y_{r}^{2}(t)-c \theta \tilde{\mathcal{L}}_{\theta}\left(Y_{l}(t), Y_{r}(t)\right) .
\end{aligned}
$$

We are in a position to define the Lyapunov function for the full state $\left(U, V, Y_{l}, Y_{r}\right)$. Let the functional $\mathcal{L}$ be defined by

$$
\forall t \geq 0, \quad \mathcal{L}\left(U(t, .), V(t, .), Y_{l}(t), Y_{r}(t)\right):=\mathcal{L}_{\theta}(U(t, .), V(t, .))+\tilde{\mathcal{L}}_{\theta}\left(Y_{l}(t), Y_{r}(t)\right) .
$$

Then the following holds.

Theorem 4. The functional $\mathcal{L}$ satisfies

$$
\frac{d}{d t} \mathcal{L} \leq\left(\tilde{C} \epsilon \frac{3+e^{\theta L}}{2}+\tilde{C}-c \theta\right) \mathcal{L} \quad \text { for a.e. } t \geq 0 .
$$

On the other hand, the best decay rate obtained by taking the minimum of the parenthesis over $\theta$ reads

$-C_{\epsilon}:=\min _{\theta \in \mathbb{R}}\left(\tilde{C} \epsilon \frac{3+e^{\theta L}}{2}+\tilde{C}-c \theta\right)=-\frac{c}{L} \ln \left(\epsilon^{-1}\right)+\left[\frac{c}{L}+\tilde{C}-\frac{c}{L} \ln \frac{2 c}{\tilde{C} L}\right]+\frac{3 \tilde{C}}{2} \epsilon \underset{\epsilon \rightarrow 0^{+}}{\sim}-\frac{c}{L} \ln \left(\epsilon^{-1}\right)$.

Proof. The estimate (3.38) follows at once from Propositions 6 and 7. On the other hand, the minimum of the function $h(\theta):=\tilde{C} \epsilon \frac{3+e^{\theta L}}{2}+\tilde{C}-c \theta$ is achieved when $e^{\theta L}=(2 c) /(\tilde{C} \epsilon L)$, which yields (3.39).

The estimate (3.38) will give the exponential decay of the $L^{2}$ norm of $(U, V)$. To derive an exponential decay for the $L^{\infty}$ norm of $(U, V)$, we need the following result.

Lemma 1. Let $u \in \operatorname{Lip}([0, L])$. Then

$$
\|u\|_{L^{\infty}(0, L)} \leq \frac{2}{\sqrt{L}}\|u\|_{L^{2}(0, L)} \quad \text { or } \quad\|u\|_{L^{\infty}(0, L)}^{3} \leq 8\|u\|_{L^{2}(0, L)}^{2}\left\|\partial_{x} u\right\|_{L^{\infty}(0, L)} .
$$

If, in addition $u(0)=0$, then

$$
\|u\|_{L^{\infty}(0, L)}^{3} \leq 16\|u\|_{L^{2}(0, L)}^{2}\left\|\partial_{x} u\right\|_{L^{\infty}(0, L)} .
$$

Proof. If $\left\|\partial_{x} u\right\|_{L^{\infty}(0, L)}=0$, then the function $u$ is constant and the first inequality in (3.40) is obvious. Assume that $\left\|\partial_{x} u\right\|_{L^{\infty}(0, L)}>0$ and pick any $x \in[0, L]$ such that

$$
u(x)=\|u\|_{L^{\infty}(0, L)} .
$$

Then we have

$$
\forall y \in[0, L], \quad|u(y)| \geq|u(x)|-\left.|| \partial_{x} u\right|_{L^{\infty}(0, L)}|y-x|,
$$

Letting

$$
D:=\frac{|u(x)|}{2\left\|\partial_{x} u\right\|_{L^{\infty}(0, L)}}=\frac{\|u\|_{L^{\infty}(0, L)}}{2\left\|\partial_{x} u\right\|_{L^{\infty}(0, L)}},
$$


we see that

$$
\forall y \in[0, L], \quad|x-y| \leq D \Rightarrow|u(y)| \geq \frac{|u(x)|}{2}=\frac{\|u\|_{L^{\infty}(0, L)}}{2} .
$$

Now if $I:=[\sup (0, x-D), \inf (L, x+D)]$, we have

$$
\|u\|_{L^{2}(0, L)}^{2} \geq \int_{I}|u(y)|^{2} d y \geq|I| \frac{\|u\|_{L^{\infty}(0, L)}^{2}}{4},
$$

and hence

$$
\|u\|_{L^{\infty}(0, L)}^{2} \leq \frac{4\|u\|_{L^{2}(0, L)}^{2}}{|I|} .
$$

From the definition of $I$ (using $x \in[0, L]$ ), we have that $|I| \geq \inf (L, D)$, so that

$$
\|u\|_{L^{\infty}(0, L)}^{2} \leq 4 \sup \left(\frac{1}{D}, \frac{1}{L}\right)\|u\|_{L^{2}(0, L)}^{2} .
$$

Using the definition of $D$, we obtain (3.40).

Assume in addition that $u(0)=0$. We claim that

$$
\|u\|_{L^{2}(0, L)}^{2} \leq 2 L^{\frac{3}{2}}\|u\|_{L^{2}(0, L)}\left\|\partial_{x} u\right\|_{L^{\infty}(0, L)} .
$$

Indeed, we have by Cauchy-Schwarz inequality

$$
u(x)^{2}-0=\int_{0}^{x} 2 u(y) \partial_{x} u(y) d y \leq 2\|u\|_{L^{2}(0, L)}\left\|\partial_{x} u\right\|_{L^{2}(0, L)} \leq 2 \sqrt{L}\|u\|_{L^{2}(0, L)}\left\|\partial_{x} u\right\|_{L^{\infty}(0, L)}
$$

and an integration w.r.t. $x \in(0, L)$ yields at once (3.42). Next, if the first estimate in (3.40) holds, taking the cube of each term and using (3.42), we arrive to (3.41). If the second estimate in (3.40) holds, then (3.41) is obvious.

Let us complete the proof of Theorem 3. Picking any $\theta \in(0,+\infty)$, we infer from (3.38) that (2.12) holds for $t \in[0,1+(1+\kappa) L / c]$ for some constant $M>0$. Increasing $M$ if needed, we see that (2.13) holds as well for $t \in[0,1+(1+\kappa) L / c]$, by using Lemma 1. Assume now that $t>1+(1+\kappa) L / c$. We pick $\theta:=L^{-1} \ln [(2 c) /(\tilde{C} \epsilon L)]$, so that, for $0<\epsilon<\epsilon_{0}$, we have $h(\theta)=-C_{\epsilon} \sim-\frac{c}{L} \ln \epsilon^{-1}$. It follows that

$$
\begin{aligned}
\int_{0}^{L}\left[U^{2}(t, x)+V^{2}(t, x)\right] d x & \leq \int_{0}^{L}\left[e^{\theta(L-x)} U^{2}(t, x)+e^{\theta x} V^{2}(t, x)\right] d x \\
& \leq e^{\theta L} \mathcal{L}(t) \\
& \leq e^{\theta L} e^{-C_{\epsilon} t} \mathcal{L}(0) \\
& \leq \frac{2 c}{\tilde{C} \epsilon L} e^{-C_{\epsilon} t}\left(\int_{0}^{L}\left[U_{0}^{2}(x)+V_{0}^{2}(x)\right] d x+\tilde{\mathcal{L}}_{\theta}\left(Y_{l}(0), Y_{r}(0)\right)\right) .
\end{aligned}
$$

But we have

$$
\left|Y_{l}(0)\right|=\left|u_{0}(0)-\bar{u}\right|=\left|u_{0}(0)-u_{\epsilon}(0)\right| \leq \delta
$$


and similarly $\left|Y_{r}(0)\right| \leq \delta$, so that

$$
\begin{aligned}
\tilde{L}_{\theta}\left(Y_{l}(0), Y_{r}(0)\right) & \leq \frac{\tilde{C}}{K(\gamma+2)} \delta^{\gamma} \exp \left(\theta \frac{c \delta^{\gamma}}{K \gamma}\right)\left\|\left(u_{0}-\bar{u}_{\epsilon}, v_{0}-\bar{v}_{\epsilon}\right)\right\|_{L^{\infty}(0, L)}^{2} \\
& \leq \frac{\tilde{C}}{K(\gamma+2)} \delta^{\gamma} \exp \left(\frac{c \delta^{\gamma}}{K L \gamma} \ln \left(\frac{2 c}{\tilde{C} \epsilon L}\right)\right)\left\|\left(u_{0}-\bar{u}_{\epsilon}, v_{0}-\bar{v}_{\epsilon}\right)\right\|_{L^{\infty}(0, L)}^{2} \\
& \leq \frac{\text { Const }}{\epsilon^{\kappa}}\left\|\left(u_{0}-\bar{u}_{\epsilon}, v_{0}-\bar{v}_{\epsilon}\right)\right\|_{L^{\infty}(0, L)}^{2}
\end{aligned}
$$

It follows that

$$
\left\|\left(u-u_{\epsilon}, v-v_{\epsilon}\right)(t)\right\|_{L^{2}(0, L)}^{2} \leq \text { Const } \frac{e^{-C_{\epsilon} t}}{\epsilon^{1+\kappa}}\left\|\left(u_{0}-\bar{u}_{\epsilon}, v_{0}-\bar{v}_{\epsilon}\right)\right\|_{L^{\infty}(0, L)}^{2} .
$$

Thus the estimate (2.12) holds for $t>1+(1+\kappa) L / c$. Finally, (2.13) follows from (2.12) and (3.40). The proof of Theorem 3 is complete.

\section{Conclusion}

In this paper, we have shown the exponential decay of the $L^{2}$-norm and of the $L^{\infty}$-norm of solutions of a quasilinear hyperbolic system of balance laws with sufficiently small source terms and appropriately chosen boundary controls. In fact, with vanishing source terms the decay rates become arbitrarily large, and thus in the limit, the case of finite-time stability is recovered. Since we work with solutions that are at each time $t \geq 0$ in the space $W^{1, \infty}(0, L)$ with respect to the space variable, the question arises whether also the $L^{\infty}$-norm of the space derivative of the solution decays exponentially. This question will be the subject of future investigations.

\section{ApPENDix}

5.1. Proof of Theorem 1. We can find some constants $N \geq 0$ and $\omega \in \mathbb{R}$ such that $\left\|e^{t A}\right\|_{\mathcal{L}(H)} \leq$ $N e^{\omega t}$ for all $t \geq 0$. On the other hand, it is well known that $A+\epsilon B$ generates also a strongly continuous semigroup in $H$. For any $u_{0} \in H$, the (mild) solution of the Cauchy problem

$$
\partial_{t} u=A u+\epsilon B u, \quad u(0)=u_{0}
$$

is denoted by $u(t)=e^{t(A+\epsilon B)} u_{0}$, and it is the solution in $C^{0}\left(\mathbb{R}^{+}, H\right)$ of the Duhamel integral equation

$$
u(t)=e^{t A} u_{0}+\int_{0}^{t} e^{(t-s) A}[\epsilon B u(s)] d s, \quad \forall t \geq 0 .
$$

Pick any $u_{0} \in H$. For given $\lambda \in(0,+\infty)$, introduce the Banach space

$$
E=\left\{u \in C^{0}\left(\mathbb{R}^{+}, H\right) ;\|u\|_{E}:=\sup _{t \geq 0}\left\|e^{\lambda t} u(t)\right\|_{H}<+\infty\right\} .
$$

For any $u \in E$, we define a function $v: \mathbb{R}^{+} \rightarrow H$ by

$$
v(t)=e^{t A} u_{0}+\int_{0}^{t} e^{(t-s) A}[\epsilon B u(s)] d s, \quad \forall t \geq 0 .
$$


Let $\Gamma(u)=v$. We aim to show that for $\epsilon \in\left(0, \epsilon_{0}\right)$ (with $\epsilon_{0}$ small enough) and $\lambda>0$ conveniently chosen, $\Gamma$ has a unique fixed-point in $E$. First, we note that $\Gamma$ maps the space $E$ into itself. Indeed, for $t \in[0, T]$ we have

$$
e^{\lambda t}\|v(t)\| \leq e^{\lambda T} N \sup \left(1, e^{\omega T}\right)\left(\left\|u_{0}\right\|+\epsilon_{0} T\|B\|_{\mathcal{L}(H)}\|u\|_{E}\right)<\infty,
$$

and for $t \geq T$, we have (using the fact that $e^{s A} w=0$ for all $s \geq T$ and all $w \in H$ )

$$
\begin{aligned}
e^{\lambda t}\|v(t)\| & \leq \int_{t-T}^{t} e^{\lambda t}\left\|e^{(t-s) A} \epsilon B u(s)\right\| d s \\
& \leq N \sup \left(1, e^{\omega T}\right) \epsilon\|B\|_{\mathcal{L}(H)}\|u\|_{E} \int_{t-T}^{t} e^{\lambda(t-s)} d s \\
& \leq N \sup \left(1, e^{\omega T}\right) \epsilon\|B\|_{\mathcal{L}(H)}\|u\|_{E} \frac{e^{\lambda T}-1}{\lambda}<\infty .
\end{aligned}
$$

Let us show now that $\Gamma$ contracts in $E$. Pick any $u_{1}, u_{2} \in E$, and let us denote $v_{1}=\Gamma\left(u_{1}\right)$, $v_{2}=\Gamma\left(u_{2}\right)$. Then we have for all $t \geq 0$

$$
\begin{aligned}
e^{\lambda t}\left\|v_{1}(t)-v_{2}(t)\right\| & \leq \int_{\sup (0, t-T)}^{t} e^{\lambda t}\left\|e^{(t-s) A} \epsilon B\left(u_{1}(s)-u_{2}(s)\right)\right\| d s \\
& \leq N \sup \left(1, e^{\omega T}\right) \epsilon\|B\|_{\mathcal{L}(H)} \frac{e^{\lambda T}-1}{\lambda}\left\|u_{1}-u_{2}\right\|_{E}
\end{aligned}
$$

so that $\Gamma$ contracts for any given $\lambda>0$ if $\epsilon \ll 1$. Then by the contraction mapping theorem, $\Gamma$ has a unique fixed-point in $E$ which is nothing but the mild solution of (5.1).

Now, pick $\lambda>0$ of the form $\lambda=C \ln \epsilon^{-1}$. Then $\Gamma$ contracts in $E$ if $k:=N \sup \left(1, e^{\omega T}\right) \epsilon\|B\|_{\mathcal{L}(H)} \frac{e^{\lambda T}}{\lambda}<$ 1 , which becomes

$$
k=\epsilon^{1-C T} \frac{N \sup \left(1, e^{\omega T}\right)\|B\|_{\mathcal{L}(H)}}{C \ln \epsilon^{-1}}<1 .
$$

This holds if $C<1 / T$ and $\epsilon \in\left(0, \epsilon_{0}\right)$ with $\epsilon_{0}>0$ small enough. On the other hand, we have that

$$
\left\|u(t)-e^{t A} u_{0}\right\|_{E} \leq(1-k)^{-1}\left\|e^{t A} u_{0}-\Gamma\left(e^{t A} u_{0}\right)\right\|_{E} \leq(1-k)^{-1} k\left\|u_{0}\right\|
$$

ant that

$$
\left\|e^{t A} u_{0}\right\|_{E} \leq N \sup \left(1, e^{\omega T}\right) e^{\lambda T} \leq \text { Const } \frac{\left\|u_{0}\right\|}{\epsilon} .
$$

It follows that $\|u\|_{E} \leq$ Const $\left\|u_{0}\right\| / \epsilon$, i.e. $\|u(t)\| \leq$ Const $e^{-\left(C \ln \epsilon^{-1}\right) t}\left\|u_{0}\right\| / \epsilon$ for all $t \geq 0$. On the other hand,

$$
\sup _{t \geq 0}\|u(t)\| \leq \sup _{t \geq 0}\left\|u(t)-e^{t A} u_{0}\right\|+\sup _{t \geq 0}\left\|e^{t A} u_{0}\right\| \leq\left[(1-k)^{-1} k+N \sup \left(1, e^{\omega T}\right)\right]\left\|u_{0}\right\| .
$$

The proof of Theorem 1 is complete. 
5.2. Proof of Theorem 2. To prove (1.5), it is sufficient to find an eigenvalue $\lambda$ of $A+\epsilon B$ of the form

$$
\lambda \sim-\frac{c}{L} \ln \epsilon^{-1} .
$$

Thus, we investigate the following spectral problem

$$
\begin{aligned}
\lambda u+c \partial_{x} u & =\epsilon v, \\
\lambda v-c \partial_{x} v & =\epsilon u, \\
u(0)=v(L) & =0 .
\end{aligned}
$$

Differentiating with respect to $x$ in (5.4), replacing $\partial_{x} v$ by its expression in (5.5) and next $v$ by its expression in (5.4), we arrive to the following ODE for $u$

$$
\partial_{x}^{2} u=\frac{\lambda^{2}-\epsilon^{2}}{c^{2}} u
$$

Similarly, we see that $v$ solves the ODE

$$
\partial_{x}^{2} v=\frac{\lambda^{2}-\epsilon^{2}}{c^{2}} v
$$

Let $\alpha \in \mathbb{C}$ be such that

$$
\alpha^{2}=\frac{\lambda^{2}-\epsilon^{2}}{c^{2}}
$$

Then $u$ and $v$ can be written as

$$
u(x)=A e^{\alpha x}+B e^{-\alpha x}, \quad u(x)=C e^{\alpha x}+D e^{-\alpha x}
$$

for some constants $A, B, C, D \in \mathbb{C}$. Plugging the expressions of $u$ and $v$ in (5.4)-(5.5) yields the system

which is satisfied if

$$
\left\{\begin{array}{l}
\lambda\left(A e^{\alpha x}+B e^{-\alpha x}\right)+c\left(A \alpha e^{\alpha x}-B \alpha e^{-\alpha x}\right)=\epsilon\left(C e^{\alpha x}+D e^{-\alpha x}\right), \\
\lambda\left(C e^{\alpha x}+D e^{-\alpha x}\right)-c\left(C \alpha e^{\alpha x}-D \alpha e^{-\alpha x}\right)=\epsilon\left(A e^{\alpha x}+B e^{-\alpha x}\right),
\end{array}\right.
$$

$$
\begin{aligned}
& (\lambda+c \alpha) A=\epsilon C, \\
& (\lambda-c \alpha) B=\epsilon D, \\
& (\lambda-c \alpha) C=\epsilon A, \\
& (\lambda+c \alpha) D=\epsilon B .
\end{aligned}
$$

Now, using (5.9), we see that (5.12) and (5.13) follow respectively from (5.14) and (5.11), so that (5.11)-(5.14) is equivalent to

$$
C=\frac{\lambda+c \alpha}{\epsilon} A, \quad B=\frac{\lambda+c \alpha}{\epsilon} D .
$$

Setting

$$
\mu=\frac{\lambda+c \alpha}{\epsilon},
$$

we wee that the solutions of (5.4)-(5.5) are the functions of the form

$$
u(x)=A e^{\alpha x}+\mu D e^{-\alpha x}, \quad v(x)=\mu A e^{\alpha x}+D e^{-\alpha x},
$$


where $A, D \in \mathbb{C}$ are arbitrary. The boundary conditions (5.6) yield the following constraints for $A$ and $D$ :

$$
\left\{\begin{array}{l}
A+\mu D=0 \\
\mu A e^{\alpha L}+D e^{-\alpha L}=0
\end{array}\right.
$$

The above system admits some nontrivial solutions if and only if the determinant of the associated matrix is null:

$$
e^{-\alpha L}-\mu^{2} e^{\alpha L}=0 .
$$

Gathering together (5.9), (5.15) and (5.16), we conclude that $\lambda$ is an eigenvalue of the operator $A+\epsilon B$ if there is some $\alpha \in \mathbb{C}$ such that

$$
\left\{\begin{array}{l}
\lambda^{2}-c^{2} \alpha^{2}=\epsilon^{2}, \\
\left(\frac{\lambda+c \alpha}{\epsilon}\right)^{2}=e^{-2 \alpha L} .
\end{array},\right.
$$

or equivalently if there are some numbers $\alpha \in \mathbb{C}$ and $s \in\{-1,1\}$ such that

$$
\begin{aligned}
\frac{\lambda+c \alpha}{\epsilon} \cdot \frac{\lambda-c \alpha}{\epsilon} & =1, \\
\frac{\lambda+c \alpha}{\epsilon} & =s e^{-\alpha L} .
\end{aligned}
$$

This yields (using $s^{-1}=s$ )

$$
\frac{\lambda-c \alpha}{\epsilon}=s e^{\alpha L} .
$$

Eliminating $\lambda$ in (5.18)-(5.19)), we conclude that system (5.17)-(5.18) is equivalent to the system

$$
\begin{aligned}
\frac{\lambda+c \alpha}{\epsilon} & =s e^{-\alpha L}, \\
\frac{\sinh (\alpha L)}{\alpha L} & =-\frac{c s}{L \epsilon} .
\end{aligned}
$$

To complete the proof, it is sufficient to pick $s=-1$ and to limit ourselves to the solutions $\alpha \in \mathbb{R}^{+}$of the equation

$$
\frac{\sinh (L \alpha)}{L \alpha}=\frac{c}{L \epsilon} .
$$

It is easily seen that the map $x \mapsto \sinh x / x$ is (strictly) increasing on $(0,+\infty)$ and onto $(1,+\infty)$, so that for any $\epsilon \in(0, c / L)$ there is a unique $\alpha=\alpha(\epsilon) \in(0,+\infty)$ satisfying (5.22). Moreover, the map $\epsilon \rightarrow \alpha$ is decreasing and $\alpha \rightarrow+\infty$ as $\epsilon \rightarrow 0^{+}$. Finally, $e^{L \alpha} /(2 L \alpha) \sim c /(L \epsilon)$ yields by taking the logarithm $L \alpha \sim \ln \epsilon^{-1}$. This gives by using again (5.22)

$$
L \alpha=\ln \epsilon^{-1}+O\left(\ln \left(\ln \epsilon^{-1}\right)\right)
$$

so that

$$
\lambda=-c \alpha+o(\epsilon)=-\frac{c}{L} \ln \epsilon^{-1}+O\left(\ln \left(\ln \epsilon^{-1}\right)\right)>-\kappa \ln \epsilon^{-1}
$$

if $\kappa>c / L$ and $0<\epsilon \ll 1$. 
5.3. Transport equation. This part follows closely the Appendix of [16].

Definition 5. Let $a=a(t, x)$ be a Lipschitz continuous function on $\mathbb{R}^{+} \times \mathbb{R}$ such that

$$
\sup _{(t, x) \in \mathbb{R}^{+} \times \mathbb{R}} \frac{|a(t, x)|}{1+|x|}<+\infty .
$$

For any pair $(t, x) \in \mathbb{R}^{+} \times \mathbb{R}$, we denote by $s \mapsto \phi_{a}(s, t, x)$ the maximal solution to the following Cauchy problem

$$
\left\{\begin{array}{l}
\dot{\theta}(s)=a(s, \theta(s)), \\
\theta(t)=x
\end{array}\right.
$$

which is defined on $\mathbb{R}^{+}$thanks to (5.23).

Proposition 8. Assume that $a=a(t, x)$ satisfies (5.23) and is of class $\mathcal{C}^{1}$ on $\mathbb{R}^{+} \times \mathbb{R}$. Then the function $\phi_{a}$ is of class $\mathrm{C}^{1}$ on $\left(\mathbb{R}^{+}\right)^{2} \times \mathbb{R}$, and we have

$$
\forall(s, t, x) \in\left(\mathbb{R}^{+}\right)^{2} \times \mathbb{R}, \quad\left\{\begin{array}{l}
\partial_{2} \phi(s, t, x)=-a(t, x) \exp \left(\int_{t}^{s} \partial_{2} a(r, \phi(r, t, x)) d r\right), \\
\partial_{3} \phi(s, t, x)=\exp \left(\int_{t}^{s} \partial_{2} a(r, \phi(r, t, x)) d r\right) .
\end{array}\right.
$$

Proof. Using the integral form of the differential equation, we obtain

$$
\forall(s, t, x) \in\left(\mathbb{R}^{+}\right)^{2} \times \mathbb{R}, \quad \phi(s, t, x)=x+\int_{t}^{s} a(r, \phi(r, t, x)) d r .
$$

We infer from the implicit function theorem (proceeding as in Section 2) that $\phi$ is of class $\mathcal{C}^{1}$ on $\left(\mathbb{R}^{+}\right)^{2} \times \mathbb{R}$. On the other hand, differentiating in (5.24) yields

$$
\forall(s, t, x) \in\left(\mathbb{R}^{+}\right)^{2} \times \mathbb{R}, \quad \partial_{2} \phi(s, t, x)=-a(t, \phi(t, t, x))+\int_{t}^{s} \partial_{2} a(r, \phi(r, t, x)) \partial_{2} \phi(r, t, x) d r .
$$

Noticing that the last equation can be viewed as a linear ODE and using the fact that $a(t, \phi(t, t, x))=a(t, x)$, we obtain the desired formula for $\partial_{2} \phi$. The other one for $\partial_{3} \phi$ is proven in the same way.

Remark 2. Is should be noted that

$$
\forall(s, t, x) \in\left(\mathbb{R}^{+}\right)^{2} \times \mathbb{R}, \quad \partial_{t} \phi(s, t, x)+a(t, x) \partial_{x} \phi(s, t, x)=0 .
$$

Definition 6. Given a positive real number $L$, we introduce for any $(t, x) \in \mathbb{R}^{+} \times[0, L]$ the sets

We also set

$$
\begin{aligned}
& F^{-}:=\{r \in[0, t] ; \forall s \in[r, t], \quad \phi(s, t, x) \in[0, L]\}, \\
& F^{+}:=\{r \in[t,+\infty) ; \forall s \in[t, r], \quad \phi(s, t, x) \in[0, L]\} .
\end{aligned}
$$

$$
e(t, x):=\inf F^{-} .
$$

Proposition 9. Assume that $a$ is a function of class $\mathrm{C}^{1}$ and that there exists $c>0$ such that

$$
\forall(t, x) \in[0,+\infty) \times[0, L], \quad a(t, x) \geq c .
$$

Then the function $e$ is of class $\mathcal{C}^{1}$ on the two open sets

$$
\begin{aligned}
G & :=\{(t, x) \in(0, T) \times(0, L) ; x<\phi(t, 0,0)\}, \\
I & :=\{(t, x) \in(0, T) \times(0, L) ; x>\phi(t, 0,0)\} .
\end{aligned}
$$


Furthermore, it holds

$$
\forall(t, x) \in G, \quad\left\{\begin{array}{l}
\partial_{t} e(t, x)=-\frac{a(t, x)}{a(e(t, x), 0)} \exp \left(-\int_{e(t, x)}^{t} \partial_{2} a(r, \phi(r, t, x)) d r\right), \\
\partial_{x} e(t, x)=-\frac{1}{a(e(t, x), 0)} \exp \left(-\int_{e(t, x)}^{t} \partial_{2} a(r, \phi(r, t, x)) d r\right),
\end{array}\right.
$$

while

$$
\forall(t, x) \in I, \quad e(t, x)=0 .
$$

The set $G$ coincide with the set of pairs $(t, x) \in(0, T) \times(0, L)$ such that

$$
e(t, x)>0 \text {. }
$$

Remark 3. The following holds

$$
\forall(t, x) \in(0, T) \times(0, L), \quad x \neq \phi(t, 0,0) \Rightarrow \partial_{t} e(t, x)+a(t, x) \partial_{x} e(t, x)=0 .
$$

Since the propagation speed $a(t, x)$ is greater than $c$, it should be clear that

$$
\forall(t, x) \in \mathbb{R}^{+} \times[0, L], \quad 0 \leq t-e(t, x) \leq \frac{L}{c} .
$$

Proof. Using (5.25), we see that for $(t, x) \in G, e(t, x)$ is the only solution (at least locally) of the equation

$$
\phi(e(t, x), t, x)=0 .
$$

An application of the implicit function theorem gives that the function $e$ is of class $\mathcal{C}^{1}$. Taking partial derivatives in (5.26) yields

$$
\partial_{t} e(t, x)=-\frac{\partial_{2} \phi(e(t, x), t, x)}{\partial_{1} \phi(e(t, x), t, x)}=-\frac{-a(t, x) \exp \left(\int_{t}^{e(t, x)} \partial_{2} a(r, \phi(r, t, x)) d r\right)}{a(e(t, x), 0)}
$$

and

$$
\partial_{x} e(t, x)=-\frac{\partial_{3} \phi(e(t, x), t, x)}{\partial_{1} \phi(e(t, x), t, x)}=-\frac{\exp \left(\int_{t}^{e(t, x)} \partial_{2} a(r, \phi(r, t, x)) d r\right)}{a(e(t, x), 0)} .
$$

Let us now consider the system

$$
\left\{\begin{array}{l}
\partial_{t} y+a(t, x) \partial_{x} y=b(t, x), \\
y(t, 0)=y_{l}(t), \\
y(0, x)=y_{0}(x)
\end{array} \quad(t, x) \in(0, T) \times(0, L) .\right.
$$

We say that a function $y \in \operatorname{Lip}([0, T] \times[0, L])$ is a strong solution of $(5.27)$ if the first equation in (5.27) holds almost everywhere and if the second and third equations in (5.27) hold everywhere. We shall need also to introduce the concept of weak solution of (5.27), following [16].

Definition 7. We say that $y \in \mathrm{C}^{0}([0, T] \times[0, L])$ is a weak solution of $(5.27)$ if for any function $\psi \in \mathcal{C}^{1}([0, T] \times[0, L])$ satisfying

$$
\begin{aligned}
\forall t \in[0, T], & \psi(t, L)=0 \\
\forall x \in[0, L], \quad \psi(T, x) & =0,
\end{aligned}
$$


it holds

$$
\begin{array}{r}
\int_{0}^{T} \int_{0}^{L}\left[y(t, x)\left(\partial_{t} \psi(t, x)+a(t, x) \partial_{x} \psi(t, x)+\partial_{x} a(t, x) \psi(t, x)\right)+b(t, x) \psi(t, x)\right] d x d t \\
+\int_{0}^{T} a(t, 0) y_{l}(t) \psi(t, 0) d t+\int_{0}^{L} y_{0}(x) \psi(0, x) d x=0 .
\end{array}
$$

Then we have the following result.

Proposition 10. 1. Let $y \in \operatorname{Lip}([0, T] \times[0, L])$. Then $y$ is a strong solution of (5.27) if and only if $y$ is a weak solution of (5.27).

2. If $a, b, y_{l}$ and $y_{0}$ are Lipschitz continuous functions, then there is at most one weak solution of (5.27).

Proof. The first assertion follows from classical arguments. The second one is proven in the Appendix of [16].

Theorem 5. Let $a, b, y_{l}$ and $y_{0}$ be functions of class $\mathcal{C}^{1}$ such that

$$
y_{l}(0)=y_{0}(0) .
$$

Then the system (5.27) admits exactly one (strong or weak) solution, and it is given explicitly by the formula

$$
\forall(t, x) \in[0, T] \times[0, L], \quad y(t, x)= \begin{cases}y_{l}(e(t, x))+\int_{e(t, x)}^{t} b(r, \phi(r, t, x)) d r & \text { if } x<\phi(t, 0,0), \\ y_{0}(\phi(0, t, x))+\int_{0}^{t} b(r, \phi(r, t, x)) d r & \text { otherwise. }\end{cases}
$$

Proof. Using Propositions 8 and 9, it is straightforward to check that the function $y$ given by formula (5.30) is in $\operatorname{Lip}([0, T] \times[0, L])$ and is of class $\mathcal{C}^{1}$, except possibly on the curve $x=\phi(t, 0,0)$ (where it is likely merely continuous), and that it is a strong solution of (5.27). On the other hand, the uniqueness of a weak solution of (5.27) follows from Proposition 10.

Corollary 1. The solution y of (5.27) satisfies the estimates:

$$
\begin{gathered}
\|y\|_{\infty} \leq \frac{L}{c}\|b\|_{\infty}+\sup \left(\left\|y_{0}\right\|_{\infty},\left\|y_{l}\right\|_{\infty}\right), \\
\left\|\partial_{x} y\right\|_{\infty} \leq \frac{1}{c} \exp \left(\frac{L}{c}\left\|\partial_{2} a\right\|_{\infty}\right) \sup \left(\|b\|_{\infty}+\left\|y_{l}^{\prime}\right\|_{\infty}+L\left\|\partial_{2} b\right\|_{\infty}, c\left\|y_{0}^{\prime}\right\|_{\infty}+L\left\|\partial_{2} b\right\|_{\infty}\right),
\end{gathered}
$$

and

$\left\|\partial_{t} y\right\|_{\infty} \leq \frac{\|a\|_{\infty}}{c} \exp \left(\frac{L}{c}\left\|\partial_{2} a\right\|_{\infty}\right) \sup \left(\|b\|_{\infty}+\left\|y_{l}^{\prime}\right\|_{\infty}+L\left\|\partial_{2} b\right\|_{\infty}, c\left\|y_{0}^{\prime}\right\|_{\infty}+L\left\|\partial_{2} b\right\| \|_{\infty}\right)+\|b\|_{\infty}$

Proof. Straightforward from (5.30).

Proposition 11. Let $(t, x) \in[0, T] \times[0, L]$ and let $\left\{a_{n}\right\} \subset \mathcal{C}^{0}([0, T] \times[0, L]) \cap L^{\infty}(0, T, \operatorname{Lip}([0,1]))$ be a sequence such that $\left\|a_{n}\right\|_{L^{\infty}(0, T, \operatorname{Lip}([0, L]))}$ is bounded and

$$
\left\|a_{n}-a\right\|_{\mathrm{e}^{0}([0, T] \times[0, L])} \rightarrow 0 \text { as } n \rightarrow+\infty,
$$


and let $\left\{\left(t_{n}, x_{n}\right)\right\} \subset[0, T] \times[0, L]$ be a sequence such that $\left(t_{n}, x_{n}\right) \rightarrow(t, x)$. Then

$$
e_{n}\left(t_{n}, x_{n}\right) \rightarrow e(t, x) \text {. }
$$

Proof. See the Appendix in [16].

Theorem 6. Let $y_{n}, a_{n}, b_{n}, y_{0, n}$ and $y_{l, n}$ be Lipschitz continuous functions such that

(i) for any $n \geq 0$, the function $y_{n}$ is a strong solution of

$$
\left\{\begin{array}{l}
\partial_{t} y_{n}+a_{n}(t, x) \partial_{x} y_{n}=b_{n}(t, x), \\
y_{n}(t, 0)=y_{l, n}(t) \\
y_{n}(0, x)=y_{0, n}(x)
\end{array} \quad(t, x) \in(0, T) \times(0, L)\right.
$$

(ii) the sequence $\left(a_{n}\right)_{n \geq 0}$ is bounded in $\operatorname{Lip}([0, T] \times[0, L])$ and it converges uniformly on $[0, T] \times[0, L]$ towards a function a;

(iii) the sequence $\left(b_{n}\right)_{n \geq 0}$ is bounded in $\operatorname{Lip}([0, T] \times[0, L])$ and it converges uniformly on $[0, T] \times[0, L]$ towards a function $b$;

(iv) the sequence $\left(y_{l, n}\right)_{n \geq 0}$ is bounded in $\operatorname{Lip}([0, T])$ and it converges uniformly on $[0, T]$ towards a function $y_{l}$;

(v) the sequence $\left(y_{0, n}\right)_{n \geq 0}$ is bounded in $\operatorname{Lip}([0, T])$ and it converges uniformly on $[0, T]$ towards a function $y_{0}$.

Then there exists a Lipschitz continuous function $y$ on $[0, T] \times[0, L]$ such that

$$
y_{n} \rightarrow y \text { in } \mathrm{C}^{0}([0, T] \times[0, L]),
$$

and $y$ is the unique solution of

$$
\left\{\begin{array}{l}
\partial_{t} y+a(t, x) \partial_{x} y=b(t, x), \\
y(t, 0)=y_{l}(t), \\
y(0, x)=y_{0}(x) .
\end{array} \quad(t, x) \in(0, T) \times(0, L) .\right.
$$

Proof. Since $y_{n}$ is the unique solution of (5.34), it follows from Corollary 1 that the sequence $\left(y_{n}\right)_{n \geq 0}$ is bounded in $\operatorname{Lip}([0, T] \times[0, L])$. From Ascoli-Arzela theorem, we can extract a subsequence $\left(y_{n_{k}}\right)_{k \geq 0}$ which converges uniformly towards a function $y$. On the other hand, since $\left(\partial_{x} a_{n}\right)_{n \geq 0}$ is bounded in $L^{\infty}((0, T) \times(0, L))$ and since the only possible weak-* limit of a convergent subsequence is $\partial_{x} a$, we have by weak-* compactness that $\partial_{x} a_{n} \rightarrow \partial_{x} a$ weakly-* in $L^{\infty}$. But this is enough to pass to the limit as $k \rightarrow+\infty$ in the weak formulation

$$
\begin{aligned}
\int_{0}^{T} \int_{0}^{L}\left[y _ { n _ { k } } ( t , x ) \left(\partial_{t} \psi(t, x)\right.\right. & \left.\left.+a_{n_{k}}(t, x) \partial_{x} \psi(t, x)+\partial_{x} a_{n_{k}}(t, x) \psi(t, x)\right)+b_{n_{k}}(t, x) \psi(t, x)\right] d x d t \\
& +\int_{0}^{T} a_{n_{k}}(t, 0) y_{l, n_{k}}(t) \psi(t, 0) d t+\int_{0}^{L} y_{0, n_{k}}(x) \psi(0, x) d x=0, \quad(5.36)
\end{aligned}
$$


where $\psi$ is any function as in Definition 7 . We arrive to

$$
\begin{aligned}
\int_{0}^{T} \int_{0}^{L}\left[y ( t , x ) \left(\partial_{t} \psi(t, x)+a(t, x)\right.\right. & \left.\left.\partial_{x} \psi(t, x)+\partial_{x} a(t, x) \psi(t, x)\right)+b(t, x) \psi(t, x)\right] d x d t \\
& +\int_{0}^{T} a(t, 0) y_{l}(t) \psi(t, 0) d t+\int_{0}^{L} y_{0}(x) \psi(0, x) d x=0,
\end{aligned}
$$

that is, $y$ is a weak solution of the transport equation (5.35). As the weak solution of (5.35) is unique by Proposition 10, we infer that there is only one possible limit for any convergent subsequence of $\left(y_{n}\right)_{n \geq 0}$, so that the whole sequence $\left(y_{n}\right)_{n \geq 0}$ converges uniformly towards $y$.

Theorem 7. Theorem 5 and Corollary 1 are still valid when we assume merely that the functions $a, b, y_{l}$ and $y_{0}$ are Lipschitz continuous.

\section{ACKNOWLEDGMENTS}

VP and LR were partially supported by the ANR project Finite4SoS (ANR-15-CE23-0007).

\section{REFERENCES}

[1] F. Alabau-Boussouira, V. Perrollaz, L. Rosier, Finite-time stabilization of a network of strings, Math. Control Relat. Fields 5 (2015), no. 4, 721-742.

[2] G. Bastin, J.-M. Coron, B. d'Andréa-Novel, On Lyapunov stability of linearized Saint-Venant equations for a sloping channel, Networks and Heterogeneous Media 4 (2009), no. 2, 177-187.

[3] F. Bouchut, A. Mangeney-Castelnau, B. Perthame, J.-P. Vilotte, A new model of Saint Venant and SavageHutter type for gravity driven shallow water flows, C. R. Math. Acad. Sci. Paris 336 (2003), no. 6, 531-536.

[4] J.-M. Coron, R. Vazquez, M. Krstic, G. Bastin, Local exponential $H^{2}$ stabilization of a $2 \times 2$ quasilinear hyperbolic system using backstepping, SIAM J. Control Optim. 51 (2013), no. 3, 2005-2035.

[5] R. Datko, J. Lagnese, M. P. Polis, An example of the effect of time delays in boundary feedback stabilization of wave equations, SIAM J. Control Optim. 24 (1986), no. 1, 152-156.

[6] J. de Halleux, C. Prieur, J.-M. Coron, B. d'Andréa-Novel, G. Bastin, Boundary feedback control in network of open channels, Automatica 39 (2003), 1365-1376.

[7] J. M. Greenberg, T.-T. Li, The effect of boundary damping for the quasilinear wave equations, J. Differential equations 52 (1984), 66-75.

[8] M. Gugat, Boundary feedbcak stabilization of the telegraph equation: Decay rates for vanishing damping term, Systems Control Lett. 66 (2014) 72-84.

[9] M. Gugat, M. Dick, G. Leugering, Gas flow in fan-shaped networks: Classical solutions and feedback stabilization, SIAM J. Control Optim., 49 (2011), 2101-2117.

[10] M. Gugat, G. Leugering, Global boundary controllability of the Saint-Venant system for sloped canals with friction, Ann. Inst. H. Poincaré Anal. Non Linéaire, 26 (2009) 257-270.

[11] M. Gugat, G. Leugering, K. Wang, Neumann boundary feedback stabilization for a nonlinear wave equation: A strict $H^{2}$-Lyapunov function, Math. Control Relat. Fields 7 (2017), no. 3, 419-448.

[12] V. Komornik, Rapid boundary stabilization of the wave equation, SIAM J. Control Optim. 29 (1991), 197-208.

[13] G. Leugering, E. J. P. G. Schmidt, On the modelling abd stabilization of flows in networks of open canals, SIAM J. Control Optim., 41 (2002), 164-180.

[14] T.-T. Li, B. Rao, Z. Wang, Exact boundary controllability and observability for first order quasilinear hyperbolic systems with a kind of nonlocal boundary conditions, Discrete Contin. Dyn. Syst. 28 (2010), 243-257.

[15] A. Majda, Disappearing solutions for the dissipative wave equation, Indiana Univ. Math. J. 24 (1974/75), 1119-1133.

[16] V. Perrollaz, Initial boundary value problem and asymptotic stabilization of the Camassa-Holm equation on an interval, J. Funct. Anal. 259 (2010), no. 9, 2333-2365. 
[17] V. Perrollaz, L. Rosier, Finite-time stabilization of hyperbolic systems over a bounded interval, in 1st IFAC workshop on Control of Systems Governed by Partial Differential Equations (CPDE2013), 2013, 239-244.

[18] V. Perrollaz, L. Rosier, Finite-time stabilization of $2 \times 2$ hyperbolic systems on tree-shaped networks, SIAM J. Control Optim. 52 (2014), no. 1, 143-163.

[19] Y. Shang, D. Liu, G. Xu, Super-stability and the spectrum of one-dimensional wave equations on general feedback controlled networks, IMA J. Math. Control and Inform. 31 (2014), 73-90.

[20] E. Zeidler, Nonlinear Functional Analysis and its Applications. I. Fixed-Point Theorems, Springer-Verlag New York Inc., 1986.

[21] G. Q. Xu, Stabilization of string system with linear bounded feedback, Nonlinear Analysis: Hybrid Systems 1 (2007) 383-397.

[22] C. Z. Xu, G. Sallet, Exponential stability and transfert functions of processes governed by symmetric hyperbolic systems, ESAIM Control Optim. Calc. Var. 7 (2002) 421-442.

Friedrich-Alexander Universität Erlangen-Nürnberg (FAU), Department Mathematik, CauerStr. 11, 91058 Erlangen, Germany

E-mail address: martin.gugat@fau.de

Laboratoire de Mathématiques et Physique Théorique, Université de Tours, UfR Sciences et Techniques, Parc de Grandmont, 37200 Tours, France

E-mail address: Vincent.Perrollaz@lmpt.univ-tours.fr

Centre Automatique et Systèmes (CAS) and Centre de Robotique (CAOR), Mines Paristech, PSL Research University, 60 Boulevard Saint-Michel, 75272 Paris Cedex 06, France

E-mail address: Lionel.Rosier@mines-paristech.fr 